\title{
The Association of Markers of Endothelial Dysfunction and Incident Diabetes Mellitus, Hypertension, Chronic Kidney Disease and Cardiovascular Mortality
}

\author{
Loretta Rena Cain \\ West Virginia University
}

Follow this and additional works at: https://researchrepository.wvu.edu/etd

\author{
Recommended Citation \\ Cain, Loretta Rena, "The Association of Markers of Endothelial Dysfunction and Incident Diabetes \\ Mellitus, Hypertension, Chronic Kidney Disease and Cardiovascular Mortality" (2011). Graduate Theses, \\ Dissertations, and Problem Reports. 3394. \\ https://researchrepository.wvu.edu/etd/3394
}

This Dissertation is protected by copyright and/or related rights. It has been brought to you by the The Research Repository @ WVU with permission from the rights-holder(s). You are free to use this Dissertation in any way that is permitted by the copyright and related rights legislation that applies to your use. For other uses you must obtain permission from the rights-holder(s) directly, unless additional rights are indicated by a Creative Commons license in the record and/ or on the work itself. This Dissertation has been accepted for inclusion in WVU Graduate Theses, Dissertations, and Problem Reports collection by an authorized administrator of The Research Repository @ WVU.

For more information, please contact researchrepository@mail.wvu.edu. 
The Association of Markers of Endothelial Dysfunction and Incident Diabetes Mellitus, Hypertension, Chronic Kidney Disease and Cardiovascular Mortality

\title{
Loretta Rena Cain
}

\author{
Dissertation submitted to the \\ School of Medicine at West Virginia University \\ in partial fulfillment of the requirements for the degree of
}

Doctor of Philosophy

in

Public Health Sciences
Anoop Shankar, MD, PhD
Alan Ductaman, MD, MSc
Jamal Mustafa, PhD
Juhua Luo, PhD
Ronald Klein MD, MPH
Daniel Sarpong, $\mathbf{P h D}$

Department of Community Medicine

Morgantown, West Virginia

2011

Keywords: Endothelial Dysfunction, Diabetes Mellitus, Hypertension, Chronic Kidney

Disease, Cardiovascular Mortality 


\section{ABSTRACT \\ The Association of Markers of Endothelial Dysfunction and Incident Diabetes Mellitus, Hypertension, Chronic Kidney Disease and Cardiovascular Mortality}

\section{Loretta R Cain}

In recent years several mechanistic animal studies have proposed new insights, including the role of endothelial dysfunction, in the pathogenesis of common cardiometabolic disorders such as diabetes mellitus, hypertension, chronic kidney disease (CKD) and cardiovascular disease (CVD). However, few studies in humans have followed up these emerging leads from animal models. Because traditional risk factors have been shown not to completely explain all the observed risk of cardiometabolic outcomes in the general population, there is a need to examine novel mechanisms such as endothelial dysfunction in population-based studies, so as to identify new ways of preventing and/or treating these diseases. In this context, we examined the novel hypothesis that higher serum levels of markers of endothelial dysfunction, including soluble vascular cell adhesion molecule-1 (sVCAM-1) and soluble intercellular adhesion molecule-1 (sICAM-1), are positively associated with the risk of developing diabetes mellitus, hypertension, CKD and cardiovascular mortality in four separate studies. We measured sVCAM1 and sICAM-1 levels from stored serum in a random sample $(\mathrm{n}=1793)$ of baseline participants from the Beaver Dam Eye Study, a population-based, longitudinal cohort study of $n=4926$ subjects ( $55 \%$ women), aged $\geq 43$ years from Beaver Dam, Wisconsin. The main outcomes of interest were 15 year incidence of diabetes mellitus, hypertension, CKD and CVD mortality occurring between the baseline examination at 1988-90 to the end of mortality follow-up at Dec 31, 2002. During the stated follow-up period, 156 subjects developed diabetes mellitus, 257 subjects developed hypertension, 269 subjects developed CKD and 212 subjects died of cardiovascular causes. We found that serum levels of sVCAM-1 and SICAM-1 are positively related to diabetes mellitus in men only (hazards ratio $[\mathrm{HR}](95 \%$ confidence interval $[\mathrm{CI}])=2.29$ [1.18-4.44] for sVCAM-1 in men), and with hypertension (HR=2.14 [1.42-3.23] for sVCAM-1) and CVD mortality $(\mathrm{HR}=1.79$ [1.17-2.76] for sICAM-1) in both men and women. In contrast, markers of endothelial dysfunction were not found to be related to $\mathrm{CKD}$. In conclusion, we have shown that serum markers of endothelial dysfunction are associated with higher risk of developing diabetes mellitus, hypertension and CVD mortality, but not with CKD. 


\section{DEDICATION}

I dedicate the completion of this dissertation to my mother, Grace Cain, who sacrificed so many things so that I could pursue my dreams. Because of her sacrifice, love and support, I have reached this milestone. Also to my 3 sisters, Betty Robinson, Lanessa Jenkins and Vanessa Wilson who were the first to pave the way for me to attend and successfully complete my academic career (at least for now). They were always there in times of struggle to ensure that my necessities were taken care of so that I could focus on my academics. There would be no success for me without them. To a very dear friend of 10 years, Donovan M Cuffie, who has seen me through life's difficulties but ever reminding me that quitting is not an option. I will always be grateful for your friendship and support. I will always be indebted to Dr. Daniel Sarpong who has been on this journey with me for almost 10 years. He has been an outstanding mentor and teacher, always encouraging me to move forward regardless the difficulty of the task, through all of the tears and disappointments, never allowing me to quit. Thank you Dr. Sarpong, for without you, I would not have accomplished. Thank you to Dr. Anoop Shankar who took me under his wing as I was extremely 'rough around the edges' and cultivated a stronger, more competent student, teacher and researcher. All of my graduate school accomplishments are because of him. Through all of these individuals, God has shown himself to me, and my faith has been refined. I will now use this milestone as a starting point to aid and speak for those who have no voice.

Proverbs 31:9 "Open thy mouth, judge righteously, and plead the cause of the poor and needy." 


\section{ACKOWLEDGEMENTS}

I would like to thank all of my dissertation committee members (Drs Anoop Shankar, Alan Ducatman, Ronald Klein, Juhua Luo, Jamal Mustafa and Daniel Sarpong) for their time and efforts to ensure that I would reach this milestone. I appreciate your expertise and hope someday to be as great as you.

I thank Dr. Jamal Mustafa for allowing me to be one his trainees, supportively funding my research and conference travels.

I thank Principal Investigators of the Beaver Dam Eye Study ( Dr. Ronald Klein and Dr. Barbara E. K. Klein) and the ancillary Beaver dam CKD Study (Dr. Ronald Klein and Dr Anoop Shankar) for allowing me to use data from their studies for this dissertation project.

I thank the staff of the Department of Community Medicine for assisting me in the day to day tasks (Leah, Claire, Alan, Colletta and Tara).

I thank all of my classmates for their friendship and collaboration. I will always be grateful. 


\section{TABLE OF CONTENTS}

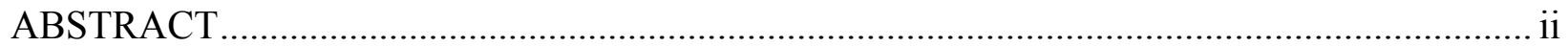

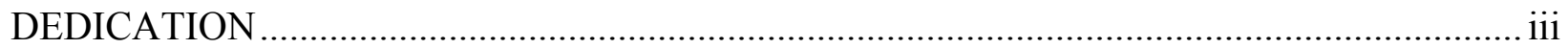

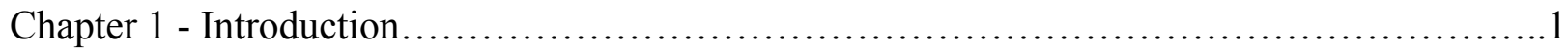

1.1 Cardiovascular Disease, Diabetes, Hypertension, CHronic kidney disease and the Need for

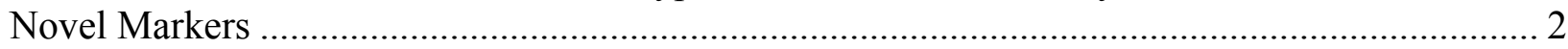

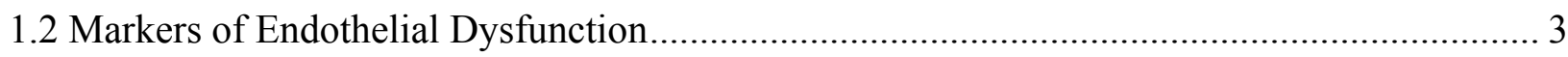

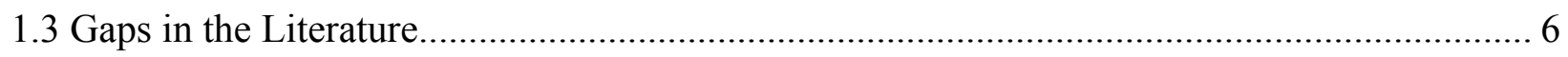

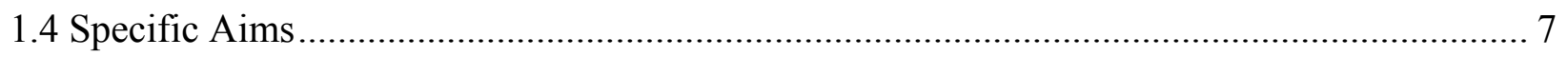

Chapter 2 - Markers of Endothelial Dysfunction and Diabetes Mellutis........................9

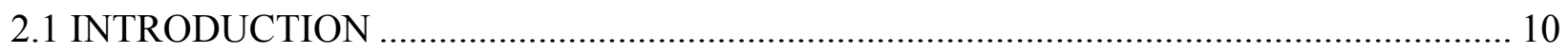

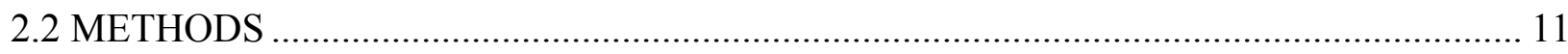

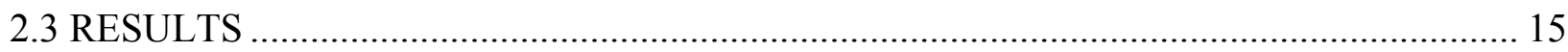

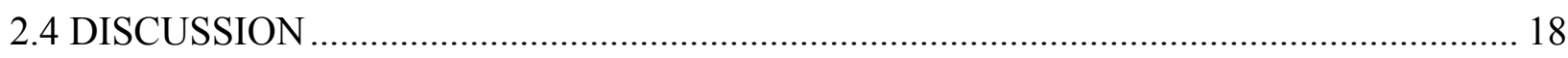

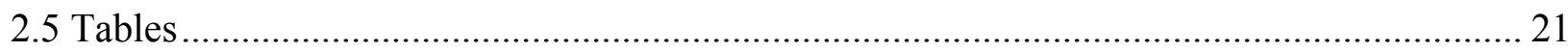

Chapter 3 - Markers of Endothelial Dysfunction and Hypertension............................25

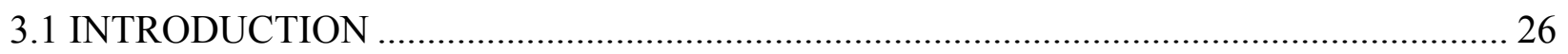

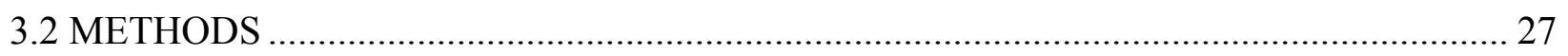

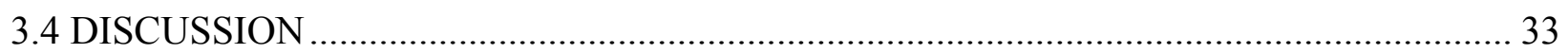

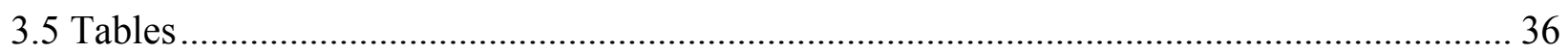

Chapter 4 - Markers of Endothelial Dysfunction and Chronic Kidney Disease..................40

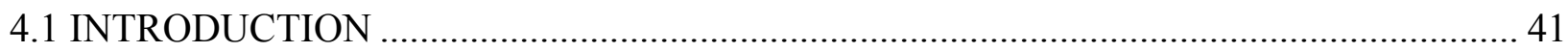

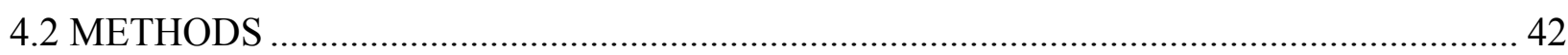

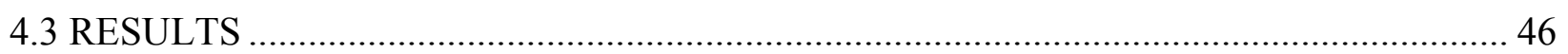

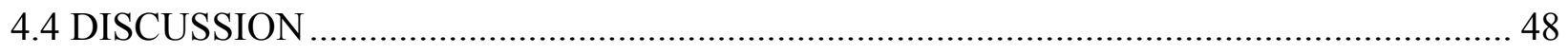

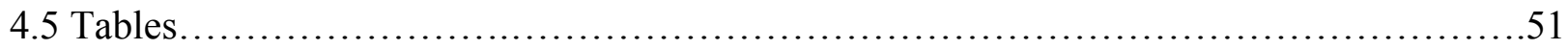

Chapter 5 - Markers of Endothelial Dysfunction and Cardiovascular Mortality..................55

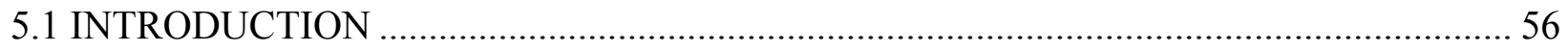

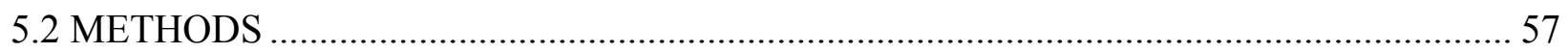




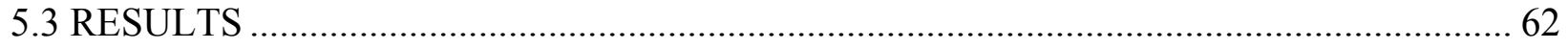

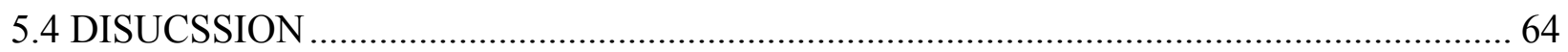

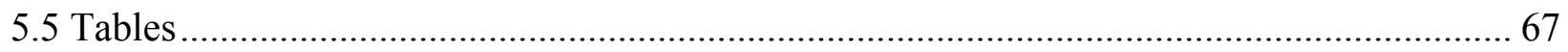

Chapter 6 - Discussion

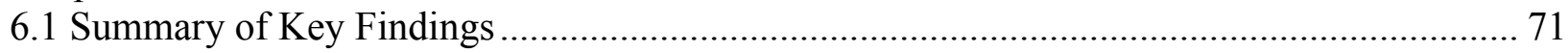

6.2 Endothelial Dysfunction and Diabetes Mellitus (Manuscript 1/Chapter 2)........................ 71

6.3 Endothelial Dysfunction and Hypertension (Manuscript 2/Chapter 3) ............................... 72

6.4 Endothelial Dysfunction and CKD (Manuscript 3/Chapter 4) .......................................... 73

6.5 Endothelial Dysfunction and Cardiovascular Mortality (Manuscript 4/Chapter 5).............. 74

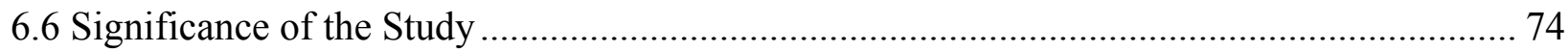

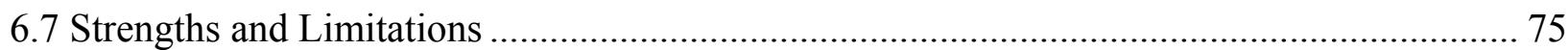

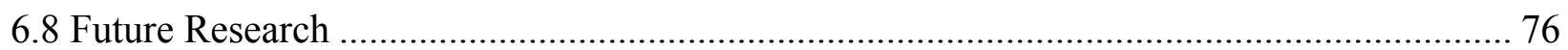

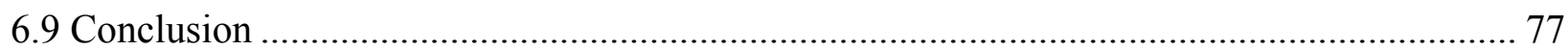

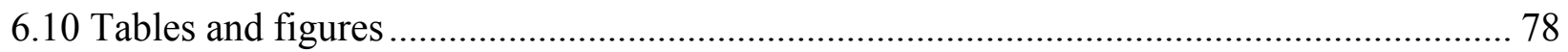

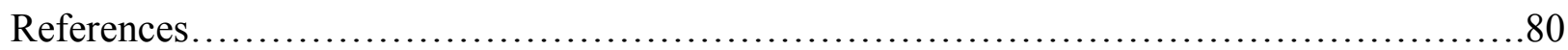


Chapter 1

\section{Introduction}




\subsection{CARDIOVASCULAR DISEASE, DIABETES, HYPERTENSION, CHRONIC KIDNEY DISEASE AND THE NEED FOR NOVEL MARKERS}

According to the Centers for Disease Control and Prevention (CDC), 2.4 million deaths occurred in the United States, with cardiovascular disease (CVD) being the number one cause of death in the United States. ${ }^{1}$ Risk factors for CVD mortality include age, ${ }^{2}$ low physical activity, ${ }^{3}$ diabetes mellitus, ${ }^{4}$ hypertension, ${ }^{5}$ coronary heart disease, ${ }^{4}$ and CKD. ${ }^{6}$ However, these traditional risk factors do not explain all the CVD risk in the general population and therefore there is a need to identify novel risk factors. In this context, mechanistic animal studies have proposed new molecular insights into the pathogenesis of CVD, such as the role of inflammation, and endothelial dysfunction. ${ }^{7}$ There is a need to examine these novel pathogenetic mechanisms in population-based studies with an aim of identifying novel predictive markers and also to advance our understanding of CVD pathogenesis in humans.

Diabetes mellitus is a known risk factor for $\mathrm{CVD},{ }^{8}$ and according to the 2007 National Diabetes Fact Sheet by CDC, 23.6 million and adults in the United States $(7.8 \%$ of the population) have diabetes. ${ }^{9}$ Known risk factors for diabetes include increasing age, ${ }^{10}$ higher body mass index (BMI) ${ }^{11}$ hypertension, ${ }^{12}$ central obesity, ${ }^{13}$ insulin resistance ${ }^{7}$ and dietary factors. ${ }^{14}$ Emerging evidence suggest that endothelial dysfunction may be a causative factor in the development of diabetes mellitus also. ${ }^{7}$

Similarly, hypertension is a known risk factor for cardiovascular disease, including myocardial infarction, heart failure and stroke. ${ }^{15}$ A recent study comparing two consecutive national surveys reported an increase in the prevalence of hypertension from $23.9 \%$ in 1988 1994 to $29.0 \%$ in $2007-2008 .{ }^{16}$ Risk factors for hypertension include a family history of

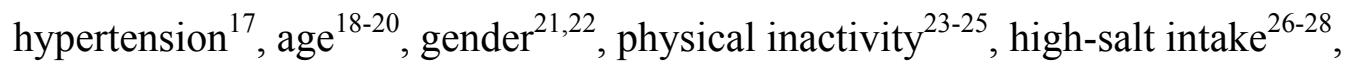


overweight/obesity ${ }^{20,29,30}$, alcohol ${ }^{20}$, smoking ${ }^{30,31}$ and diabetes mellitus ${ }^{15,19,32}$ However, these traditional risk factors may not account for all the observed risk of hypertension in the general population. ${ }^{33}$ In this context, based on predominantly data from animal studies,${ }^{34}$ endothelial dysfunction is now being proposed as a novel mechanism that may be involved in the development of hypertension.

Chronic kidney disease (CKD) is recognized as a strong risk factor for incident $\mathrm{CVD}^{35}$, including coronary artery disease and stroke, and it also independently predicts CVD mortality. ${ }^{36}$ Identifying risk factors for $\mathrm{CKD}$ are therefore important in preventing $\mathrm{CVD} .{ }^{35} \mathrm{~A}$ recent study comparing two consecutive national surveys reported an increase in the prevalence of CKD between 1988-1994 and 1999-2004 suggesting it to be a growing public health problem. ${ }^{37}$ Diabetes mellitus ${ }^{38,39}$ and hypertension ${ }^{39-44}$ have been consistently shown to be important risk factors in the development of kidney disease. Studies have shown that several of the known cardiovascular risk factors are also related to the development of $\mathrm{CKD}$, including advancing age,${ }^{38,39,45,46}$ male sex,${ }^{39-44}$ obesity, ${ }^{39-44,47}$ smoking,,${ }^{39,44,48}$ high triglycerides, ${ }^{49}$ low high-density lipoprotein (HDL) cholesterol, ${ }^{39,49,50}$ and a mild reduction in GFR at baseline. ${ }^{39}$ However, these traditional risk factors do not account for all the observed CKD risk in the general population..$^{39,51,52}$ Therefore studies are needed to examine the role of new predictors in the development of $\mathrm{CKD}$, including markers of endothelial dysfunction.

\subsection{MARKERS OF ENDOTHELIAL DYSFUNCTION}

The vascular endothelium is involved in a variety of key processes important to human health and disease, including control of coagulation, ${ }^{53}$ fibrinolysis. ${ }^{53,54}$ vascular tone ${ }^{53,55}$ and growth, ${ }^{55}$ immune response ${ }^{53,55}$ and oxidative stress. ${ }^{54,56}$ Endothelial dysfunction has been found 
to be closely related to insulin resistance, ${ }^{7,57}$ elevated levels of inflammatory markers, ${ }^{7,58}$ impaired function of lipoprotein lipase (contributing to high triglycerides and low HDL cholesterol levels, ${ }^{7,58}$ impaired nitric oxide release ${ }^{58}$ and endothelium dependent vasodilation. ${ }^{58}$

Studies suggest that endothelial dysfunction is associated with risk factors and processes associated with diabetes mellitus, such as hyperglycemia, ${ }^{59}$ inflammation, ${ }^{59}$ and insulin resistance. ${ }^{7,57}$ One of the well-known vasodilators produced in the endothelium is nitric oxide (NO) whose production is decreased due to endothelial dysfunction, ${ }^{60}$ resulting from reduced activity of endothelial NO (eNOS). Early stages of endothelial dysfunction are related to increased production of reactive oxygen species (ROS) which in turn can cause more damage to endothelial and vascular function. ${ }^{61}$ ROS are now considered to be a major risk factor in the development of insulin resistance, diabetes mellitus, hypertension, and CVD. ${ }^{62}$

Endothelial dysfunction may cause hypertension by impairing the vasodilator mechanism of NO (which is also called endothelium-derived relaxing factor), subsequently causing elevated blood pressure. ${ }^{63}$ Endothelial dysfunction may also be related to other mechanisms involved in the development of hypertension, including insulin resistance ${ }^{64}$, systemic inflammation ${ }^{65}$ and hyperuricemia. ${ }^{66}$

Endothelial dysfunction is present in end-stage renal disease. ${ }^{67,68}$ Biomarkers of endothelial dysfunction, including circulating adhesion molecules VCAM-1, ICAM-1, and Eselectin are shown to be elevated even in less advanced stages of kidney disease. ${ }^{67,69,70}$ Endothelin-1 (ET-1), an endothelium-derived growth factor, of both renal mesangial and extrarenal vascular origin and its interaction with angiotensin-II are implicated in the progression of renal disease. ${ }^{71}$ For example, Koobi et al. ${ }^{72}$ showed that Angiotensin II type 1 (AT1) receptor blockade in nephrectomized rats significantly reduced the vasoconstriction response in relation 
to Endothelin-1 administration. Angiotensin-II, a central molecule in the initiation and progression of chronic renal disease $\mathrm{e}^{49,73}$ is closely related to endothelial function and inflammation ${ }^{74}$ and treatment with angiotensin converting enzyme inhibitors have been shown to lower circulating biomarker levels of inflammation and endothelial dysfunction ${ }^{75}$. These findings suggest that endothelial dysfunction may precede and can predict the development of CKD.

Animal studies show that endothelial dysfunction may have a causative role in the development of cardiovascular disease. ${ }^{76}$ The intact, healthy endothelium secretes various cardio-protective substances including nitric oxide, which diffuses to surrounding tissues and cells, relaxing smooth-muscle cells and preventing platelet adhesion and aggregation, preventing expression of adhesion molecules, leukocyte adhesion and migration into the arterial wall and arterial smooth muscle cell proliferation ${ }^{77}$. Conversely, early stages of endothelial dysfunction is characterized by increased formation of reactive oxygen species and increased expression of adhesion molecules such as SVCAM-1 and sICAM-1, which can react with NO forming peroxynitrate $^{78}$, reducing NO bioavailability, which over the long run may be involved in increased risk of cardiovascular disease ${ }^{79}$.

Because most methods of assessing endothelial dysfunction are invasive and are not suitable for large-scaled epidemiological studies, ${ }^{80}$ circulating levels of cellular adhesion molecules such as such as intercellular adhesion molecule 1 (ICAM-1), and vascular cell adhesion molecule 1 (VCAM-1) are highly specific and valid markers of atherosclerosis-related endothelial dysfunction and have been used extensively in epidemiologic studies that utilized stored biospecimen ${ }^{81,82}$ 


\subsection{GAPS IN THE LITERATURE}

The few previous studies exploring the relationship between endothelial dysfunction and diabetes mellitus have reported contrasting relationships by gender, with some studies finding an association only in men ${ }^{83,84}$ and others in women. ${ }^{81}$ Therefore the relationship between endothelial dysfunction and diabetes mellitus by gender remains unclear. In the current project, we will examine this association using serum markers of endothelial dysfunction, including sVCAM-1 and sICAM-1 in the Beaver Dam Eye Study, a large population-based cohort from Wisconsin which included men and women in approximately equal number.

Previous studies exploring the relationship between endothelial dysfunction and hypertension have found positive relationships in specialized populations such as pregnant women, ${ }^{85}$ subjects with type 1 diabetes, ${ }^{86}$ and those with obstructive sleep apnea-hypopnea syndrome. ${ }^{87}$ In the general population, only one previous cross-sectional study from China have examined this association. ${ }^{88}$ Even though they reported a positive association between serum sVCAM-1 and sICAM-1 levels and prevalent hypertension, due to the cross-sectional nature of the study, the authors could not clarify whether adhesion molecules were elevated prior to hypertension development or not. Therefore, a longitudinal cohort study exploring the relationship between endothelial dysfunction in the general population is needed. In this context, we will prospectively examine this association using markers of endothelial dysfunction in the Beaver Dam Eye Study, a large, population-based, longitudinal cohort study from Wisconsin.

Previous cross-sectional studies exploring the relationship between endothelial dysfunction and CKD found positive associations. ${ }^{67,69,70}$ However, endothelial function markers are elevated as a consequence of CKD and dialysis. ${ }^{89,90}$ Therefore, a longitudinal study is necessary to determine whether or not endothelial dysfunction preceded CKD onset. In the 
current project, we will measure serum markers of endothelial dysfunction, including sVCAM-1 and sICAM-1, from stored serum specimens at the baseline examination and examine its relationship to the risk of developing CKD over a 15 year follow-up period.

Previous studies have reported associations between markers of endothelial dysfunction and cardiovascular mortality in ill populations, including those with kidney disease ${ }^{91}$, heart failure $^{92}$ and arthritis ${ }^{93}$. However, to our knowledge, no previous study has examined the association between markers of endothelial dysfunction and cardiovascular mortality in the general population. Therefore, we will use markers of endothelial dysfunction to explore cardiovascular mortality in a non-clinical population.

\subsection{SPECIFIC AIMS}

The primary objectives of the current dissertation project are to determine the relations of biomarkers of endothelial dysfunction to the long-term development of diabetes, hypertension, chronic kidney disease and cardiovascular mortality in the general population. The proposal takes advantage of the data on exposures and outcomes, including mortality, gathered over the last 15 years, from a population-based cohort in Beaver Dam, Wisconsin. In the Beaver Dam Eye Study (BEDS), we have data on glucose, glycosolated hemoglobin, and insulin injection use, blood pressure and serum creatinine measured at the baseline, 5-year, 10-year and 15-year follow-up examinations and mortality data which will enable us to define our primary outcomes, diabetes, hypertension, CKD, cardiovascular (CVD) mortality and all-cause mortality. We also have markers of endothelial dysfunction, including sVCAM-1 and sICAM-1 levels already measured from the baseline blood samples of the cohort as part of a previous ancillary study. Our study provides a unique opportunity to assess the temporal associations between new risk factors, 
such as markers of endothelial dysfunction, and the development of diabetes, hypertension, CKD and mortality over a period of 15 years.

The specific aims of the current study are to determine the relation between biomarkers of endothelial dysfunction (intercellular adhesion molecule 1 [ICAM-1] and vascular cell adhesion molecule 1 [VCAM-1]) and the risk of developing

1) diabetes mellitus;

2) hypertension;

3) $\mathrm{CKD}$; and

4) CVD mortality over a 15 -year follow-up period. 
Chapter 2

Manuscript 1: The Association of Markers of Endothelial Dysfunction and Incident Diabetes 


\subsection{INTRODUCTION}

Endothelial dysfunction is a key pathophysiological process involved in the development of cardiovascular disease. ${ }^{94}$ Endothelial dysfunction has also been shown to be related to insulin resistance $^{95}$, hyperglycemia ${ }^{59}$ and inflammation ${ }^{59}$ and therefore may have a role in the development of diabetes mellitus. Also, in subjects with diabetes, endothelial dysfunction has been reported to be predictive of subsequent complications, including nephropathy, retinopathy, and cardiovascular disease. ${ }^{96}$ Soluble adhesion molecules, including serum levels of vascular cell adhesion molecule-1 (sVCAM-1) and intercellular adhesion molecule -1 (sICAM-1) have been used as markers of endothelial dysfunction in epidemiological studies. ${ }^{97}$ However, few epidemiological studies have examined the relationship between soluble adhesion molecules and incident diabetes and the results have not been consistent, as some studies reported a positive association ${ }^{98-100}$ while others did not. ${ }^{84,101}$ Therefore, the independent relationship between sVCAM-1 and sICAM-1 and incident diabetes in the general population remains unclear. Therefore, we examined serum levels of sVCAM-1 and sICAM-1 in relationship to incident diabetes in a population-based sample of adults from Wisconsin. 


\subsection{METHODS}

\subsubsection{Population}

The current study is based on date from the Beaver Dam Eye study, a population-based cohort study in Wisconsin originally aimed at studying age-related eye diseases. The methods used to identify and describe the Beaver Dam population have appeared in previous reports. ${ }^{102,103}$ In brief, a private census of the population of Beaver Dam, Wisconsin, was performed from September 1987 to May 1988 to identify all residents in the city or township of Beaver Dam who were 43-84 years of age. Of the 5,924 eligible individuals (98\% Caucasians), 4,926 (83.1\%) participated in the baseline examination between March 1, 1988 and September 14, 1990. Comparisons between participants and nonparticipants at the time of the baseline examination have appeared elsewhere. ${ }^{103}$

The baseline examination was followed by a 5-year follow-up examination from 1993 to 1995, a 10-year follow-up examination from 1998-2000, and a 15-year follow-up examination from 2003-2005. Written informed consent was obtained from each subject at each examination. The study was approved by the Human Subjects Committee of the University of Wisconsin School of Medicine and Public Health, Madison, WI. Levels of sVCAM-1 and SICAM-1 were measured in stored frozen serum on a random sample of the baseline cohort $(\mathrm{n}=1793)$ as part of an ancillary study examining risk factors for kidney disease.

For the longitudinal analyses with sVCAM-1 and sICAM-1 as the main exposure, out of 1793 individuals with available sICAM-1 and sVCAM-1 measurements who participated in the baseline and at least one follow-up examination, we excluded subjects with diabetes mellitus at baseline ( $\mathrm{n}=84)$, missing information on covariates included in the multivariable model $(\mathrm{n}=326)$ and those who had a history of cardiovascular disease $(n=264)$. This resulted in $n=1119$ diabetes- 
free individuals with biomarker complete covariate information to form the at-risk study population, among whom $n=156$ developed incident diabetes over the 15 -year follow-up period.

\subsubsection{Exposure ascertainment}

The baseline and follow-up examinations included measurement of weight, height, systolic and diastolic blood pressure by trained observers and administering a standardized questionnaire that collected information regarding participants' demographic characteristics, details regarding cigarette smoking, alcohol intake, medical histories and medications taken, including physician-diagnosed diabetes, hypertension or cardiovascular disease (CVD). Nonfasting blood specimens were obtained for measurement of plasma glucose, glycosylated hemoglobin, serum total cholesterol and high density lipoprotein (HDL) cholesterol.

Age was defined as the participants' age at the time of the baseline examination.

Education was categorized as less than high school, high school, or beyond high school. Body mass index (BMI) was defined as participants' weight in kilograms divided by their height in meters squared. Hypertension was defined as a systolic blood pressure of $140 \mathrm{mmHg}$ or higher, and/or a diastolic blood pressure $90 \mathrm{mmHg}$ or higher, and/or the combination of self-reported hypertension diagnosis by a physician and use of antihypertensive medications.

At cohort examinations, blood samples were collected in three 10-mL liquid EDTA blood tubes, placed on ice packs, stored in Styrofoam containers until they were centrifuged and aliquoted for storage in freezers at $-80^{\circ} \mathrm{C}$ until the time of laboratory analysis. Quality control samples were routinely frozen with study participant samples. Baseline frozen plasma samples were randomly ordered (to further reduce systematic bias and inter assay variation) and thawed and batch-analyzed for markers of endothelial function (sICAM-1, sVCAM-1). 
sICAM-1 (soluble intercellular adhesion molecule-1) was measured in plasma using the quantitative sandwich enzyme technique of the enzyme-linked immunosorbent assay (ELISA) Parameter kit from R \& D Systems (Minneapolis, MN). The intensity of the color was measured on a SpectraMax spectrophotometer (Molecular Devices, Sunnyvale, California). The interassay $\mathrm{CV}$ range was $6.0-10.1 \%$ for the plasma assay.

sVCAM-1 (soluble vascular cell adhesion molecule-1) was measured in plasma or serum using the quantitative sandwich enzyme technique of the enzyme-linked immunosorbent assay (ELISA) Parameter kit from R \& D Systems (Minneapolis, MN). The intensity of the color was measured on a SpectraMax spectrophotometer (Molecular Devices, Sunnyvale, California). The inter-assay $\mathrm{CV}$ range was $8.9-10.2 \%$ for the plasma assay.

\subsubsection{Outcome of Interest: Diabetes Mellitus}

Persons were defined as having diabetes mellitus if were treated with insulin, oral hypoglycemic agents, or were newly classified as having diabetes based on the glycosylated hemoglobin value that was $\geq 6.5 \%$, consistent with recent American Diabetes Association guidelines $^{104}$. Incident diabetes was defined as having newly diagnosed diabetes at the 5-, 10-, or 15-year follow-up examination among subjects free of diabetes at baseline.

\subsubsection{Statistical methods}

We were interested in the association between serum levels of SVCAM-1 and SICAM-1 and 15-year incident diabetes. We categorized VCAM and ICAM into tertiles for the main analysis. 
We used chi-square test and analysis of variance to compare the relationship of selected baseline characteristics to increasing categories of VCAM. We used multivariable Cox proportional hazard regression models to determine the hazard ratio (HR) and 95 percent confidence interval (CI) of 15-year incident diabetes, controlling simultaneously for potential confounders. We used a multivariable-adjusted Cox proportional hazard regression model, adjusted for age, gender (female, male), education ( $<$ high school, high school, $>$ high school), smoking (never, former, current), alcohol intake (never, former, current), BMI $\left(\mathrm{kg} / \mathrm{m}^{2}\right)$, hypertension (absent, present), mean arterial blood pressure (mm Hg), serum total cholesterol (mg/dL) and high sensitivity Creactive protein $(\mathrm{mg} / \mathrm{dL})$. To examine the consistency of the association between sVCAM-1 and sICAM-1 levels and incident diabetes, we performed subgroup analyses by gender (men, women) and hypertensive status (yes, no). 


\subsection{RESULTS}

Among 1119 study participants included in the current analysis, there were 472 men and 647 women. Overall, 156 subjects (13.9\%) developed diabetes over an average 15-year followup period, including 83 men and 73 women.

Table 2.1 presents the characteristics of the study population by increasing serum sVCAM-1 levels at baseline. Those who had higher serum sVCAM-1 levels were more likely to be older, educated below high school, never smokers and never or former drinkers. They were also likely to have hypertension, lower total serum cholesterol levels and higher systolic blood pressure.

Table 2.2 presents the association between increasing categories of serum sVCAM-1, sICAM-1 and 15-year incident diabetes. The multivariable model for sVCAM-1 showed a positive association between increasing serum sVCAM-1 levels and incident diabetesSimilarly, the multivariable model for sICAM-1 showed a positive association between increasing serum sICAM-1 levels and incident diabetes and the model evaluating linear trend in this association was similarly just shy of statistical significance $(\mathrm{p}$-trend $=0.0623)$.

Table 2.3 presents the associations between increasing categories of serum sVCAM-1, sICAM-1 and 15-year incident diabetes, stratified by gender. The multivariable model for sVCAM-1 in women showed no association between increasing serum sVCAM-1 levels and incident diabetes. In contrast, the multivariable model for sVCAM-1 in men showed a positive association between increasing serum sVCAM-1 levels and incident diabetes. The model evaluating the trend was statistically significant. Similarly, the multivariable model for sICAM-1 in women showed no association between increasing serum sICAM-1 levels and incident diabetes. In contrast, the multivariable model for sICAM-1 in men showed a positive association 
between increasing sICAM-1 levels and incident diabetes. The model evaluating the trend was statistically significant.

Table 2.4 presents the associations between increasing categories of serum sVCAM-1, sICAM-1 and 15-year incident diabetes, stratified by hypertension status. The multivariable model for sVCAM-1 in those without hypertension showed no association between increasing serum sVCAM-1 levels and incident diabetes. In contrast, the multivariable model for sVCAMlin those with hypertension showed a positive association between increasing serum sVCAM-1 levels and incident diabetes. The model evaluating the trend was just shy of statistical significance. For sICAM-1, a similar pattern of association with incident diabetes was observed, with no association among those without hypertension and a positive association among those with hypertension. The model evaluating the trend was statistically significant.

Supplementary analyses were conducted to examine the association between serum sVCAM-1 and sICAM-1 and incident diabetes, stratified by obesity status. We found that serum sVCAM-1 and sICAM-1 were related to incident diabetes both among non-obese and obese subjects. Compared to the lowest tertile of sVCAM-1 (referent), the multivariable odds ratio (95\% confidence interval) of incident diabetes was $1.45(0.79,2.66)$ in tertile 2 and $1.97(1.06-$ $3.67)$ in tertile 3 in obese subjects, and $1.01(0.50,2.01)$ in tertile 2 and $1.60(0.88-2.90)$ in tertile 3 in non-obese subjects; $p$-interaction=0.1452. Results were similar for sICAM-1.

A second set of supplementary analyses were conducted to examine the association between serum sVCAM-1 and sICAM-1 and incident diabetes, additionally adjusted for baseline glycosolated hemoglobin in the multivariable model. Compared to the lowest tertile of sVCAM1 (referent), the multivariable odds ratio ( $95 \%$ confidence interval) of incident diabetes was 1.59 $(1.03,2.46)$ in tertile 2 and $1.67(1.04-2.68)$ in tertile 3. Compared to the lowest tertile of 
sICAM-1 (referent), the multivariable odds ratio (95\% confidence interval) of incident diabetes was $1.29(0.62,2.68)$ in tertile 2 and $1.67(1.00-2.79)$ in tertile 3. 


\subsection{DISCUSSION}

In a population-based sample of adults from Wisconsin, increasing serum levels of sVCAM-1 and sICAM-1 were found to be positively associated with incident diabetes mellitus in men, and in subjects with hypertension. In contrast, serum levels of sICAM-1 and sVCAM-1 were not found to be associated with diabetes mellitus in women, or in subjects without hypertension. These contrasting associations were independent of smoking, alcohol intake, education, hypertension, body mass index, total cholesterol and high sensitivity $\mathrm{C}$ - reactive protein levels.

Serum levels of sICAM-1 and sVCAM-1 have been used in epidemiological research before as reliable markers of endothelial dysfunction. ${ }^{97}$ Previous studies have reported that markers of endothelial dysfunction are associated with increased risk of developing cardiovascular disease. ${ }^{94}$ As diabetes is one of the strong risk factors for cardiovascular disease, we examined if endothelial dysfunction markers are also related to diabetes mellitus. Subsequently we found that markers of endothelial dysfunction are related to diabetes in men and subjects with hypertension.

Our study results of an association between markers of endothelial dysfunction and diabetes in men are consistent with a previous study by Thorand et al. showing serum sICAM-1 levels to be associated with increased risk of type 2 diabetes in men and not in women, with hazard ratios of 1.32 and 1.03 , respectively. ${ }^{84}$ Also, another study showed that when measuring endothelial function as reactive hyperemia by peripheral arterial tonometry, endothelial dysfunction was associated in adolescent males but not adolescent females. ${ }^{83}$ However, our findings are inconsistent with previous research reporting sVCAM-1 and sICAM-1 to be associated with diabetes in elderly women. ${ }^{98-100}$ 
Several metabolic abnormalities associated with diabetes mellitus are thought to contribute to endothelial dysfunction by disrupting mechanisms that regulate nitric oxide synthesis, including conditions such as hyperglycemia, insulin resistance, excess free fatty acids release and compensatory hyperinsulinemia. ${ }^{105}$ Therefore, it would be plausible for diabetes mellitus to be associated with endothelial dysfunction.

The correct explanation for the lack of association between markers of endothelial dysfunction and diabetes in women in the current study is not clear. One explanation may be that female hormones are cardioprotective and therefore to some extent may mitigate any adverse effect of endothelial dysfunction. ${ }^{106}$ There is a need for more studies in this topic to clarify the modifying effect of female gender in the association between endothelial dysfunction and diabetes.

In the current study we found that markers of endothelial dysfunction are associated with diabetes among subjects with hypertension. It has been shown that endothelial dysfunction is one of the hallmarks of hypertension ${ }^{107-109}$. Diabetes and hypertension are known to occur together as part of the insulin resistance syndrome ${ }^{110}$ and as per the "common soil" hypothesis, ${ }^{111}$ as they may share common antecedents, such as adverse environmental conditions and less than optimal nutrition. A corollary observation to our findings is that along with insulin resistance, endothelial dysfunction may be one of the antecedent factors shared by hypertension and diabetes mellitus.

Strengths of this study include its population-based sample, high participation rate, use of standardized protocols for exposure and outcome measurement and the availability of specific markers of endothelial dysfunction, including sVCAM-1 and sICAM-1 levels. Also, misclassification of diabetes status may have biased our results. A main study limitation is the generalizability of our study to other populations. 
In conclusion, in a population-based sample of Wisconsin adults, we found that increasing serum levels of sVCAM-1 and sICAM-1 levels were positively associated with 15year incident diabetes mellitus in men and in subjects with hypertension, independent of age smoking status, alcohol intake, education, hypertension, BMI, total cholesterol levels and high sensitivity c-reactive protein levels. In contrast, serum levels of sICAM-1 and sVCAM-1 were not found to be associated with diabetes mellitus in women, or in subjects without hypertension. This finding may suggest sVCAM-1 and sICAM-1 to be a novel risk factor risk factor in the development of diabetes mellitus in men and in those with hypertension. 


\subsection{TABLES}

Table 2.1. Baseline characteristics by vascular cell adhesion molecule 1 (sVCAM -1) tertiles

\begin{tabular}{|c|c|c|c|c|}
\hline \multirow[b]{2}{*}{ Characteristics } & \multicolumn{3}{|c|}{ Serum SVCAM-1 tertiles* } & \multirow[b]{2}{*}{ p-value $\dagger$} \\
\hline & Tertile 1 & Tertile 2 & Tertile 3 & \\
\hline Age, years & $55.6 \pm 0.51$ & $58.40 \pm 0.51$ & $63.49 \pm 0.51$ & $<0.0001$ \\
\hline Females, $\%$ & $56.2 \%$ & $58.2 \%$ & $59.1 \%$ & 0.7243 \\
\hline Education, \% & & & & $<0.0001$ \\
\hline Below high school & $14.6 \%$ & $18.2 \%$ & $27.8 \%$ & \\
\hline High school & $47.3 \%$ & $48.6 \%$ & $43.6 \%$ & \\
\hline Above high school & $38.1 \%$ & $33.2 \%$ & $28.6 \%$ & \\
\hline Smoking, $\%$ & & & & 0.1765 \\
\hline Never & $43.5 \%$ & $44.8 \%$ & $49.6 \%$ & \\
\hline Former & $32.4 \%$ & $33.4 \%$ & $33.3 \%$ & \\
\hline Current & $24.1 \%$ & $21.7 \%$ & $17.1 \%$ & \\
\hline Alcohol intake categories, $\%$ & & & & 0.0062 \\
\hline Never & $1.1 \%$ & $3.0 \%$ & $4.7 \%$ & \\
\hline Former & $5.7 \%$ & $7.3 \%$ & $7.9 \%$ & \\
\hline Current drinker, $<2$ drinks/day & $81.4 \%$ & $81.3 \%$ & $81.6 \%$ & \\
\hline Current drinker, $\geq 3$ drinks/day & $11.9 \%$ & $8.4 \%$ & $5.8 \%$ & \\
\hline Body mass index (BMI), $\mathrm{kg} / \mathrm{m}^{2}$ & $28.4 \pm 0.28$ & $28.2 \pm 0.28$ & $28.9 \pm 0.28$ & $<0.0001$ \\
\hline Serum total cholesterol $\mathrm{mg} / \mathrm{dL}$ & $241.5 \pm 2.25$ & $232.5 \pm 2.26$ & $226.1 \pm 2.22$ & $<0.0001$ \\
\hline Hypertension, $\%$ & $39.2 \%$ & $38.6 \%$ & $52.0 \%$ & 0.0002 \\
\hline Systolic blood pressure, $\mathrm{mm} \mathrm{Hg}$ & $127.5 \pm 0.98$ & $127.7 \pm 0.98$ & $133.1 \pm 0.97$ & $<0.0001$ \\
\hline Diastolic blood pressure, $\mathrm{mm} \mathrm{Hg}$ & $79.1 \pm 0.54$ & $77.3 \pm 0.54$ & $77.9 \pm 0.53$ & $<0.0001$ \\
\hline
\end{tabular}


Table 2.2. Association between increasing serum levels of sVCAM-1 and sICAM-1 and incident diabetes

\begin{tabular}{|c|c|c|c|}
\hline $\begin{array}{l}\text { Serum sVCAM-1 tertiles* } \\
(\mathrm{n}=1119)\end{array}$ & $\begin{array}{l}\text { No. at } \\
\text { risk }\end{array}$ & Cases & $\begin{array}{l}\text { Multivariable-adjusted } \\
\text { hazards ratio }(95 \% \text { confidence interval })\end{array}$ \\
\hline Tertile 1 & 370 & 42 & 1 (referent) \\
\hline Tertile 2 & 368 & 60 & $1.63(1.07,2.49)$ \\
\hline Tertile 3 & 381 & 54 & $1.46(0.93,2.31)$ \\
\hline p-trend & & & 0.1187 \\
\hline \multicolumn{4}{|l|}{$\begin{array}{l}\text { Serum sICAM-1 tertiles } \dagger \\
(n=1119)\end{array}$} \\
\hline Tertile 1 & 370 & 43 & 1 (referent) \\
\hline Tertile 2 & 368 & 48 & $1.09(0.71,1.69)$ \\
\hline Tertile 3 & 381 & 65 & $1.52(0.99,2.33)$ \\
\hline p-trend & & & 0.0528 \\
\hline \multicolumn{4}{|c|}{$\begin{array}{l}\text { *sVCAM-1 Tertiles :Tertile } 1(\leq 682.2 \mathrm{ng} / \mathrm{ml}) \text { Tertile } 2(682.3-826.2 \mathrm{ng} / \mathrm{ml}) \text { Tertile } 3(\geq 826.2 \mathrm{ng} / \mathrm{ml}) \\
\dagger \text { sICAM-1 Tertiles : Tertile } 1(\leq 247.5 \mathrm{ng} / \mathrm{ml}) \text { Tertile } 2(247.6-302.2 \mathrm{ng} / \mathrm{ml}) \text { Tertile } 3(\geq 302.2 \mathrm{ng} / \mathrm{ml}) \\
\text { \$Adjusted for age (years), sex }(\text { male, female), education categories }(<\mathrm{high} \text { school, high school, }>\text { high school }) \text {, } \\
\text { smoking (never, former, current), alcohol intake (never, former, current), body mass index }\left(\mathrm{kg} / \mathrm{m}^{2}\right) \text {, hypertension } \\
\text { (absent, present) and serum cholesterol }(\%) \text {, high sensitivity C reactive protein }(\mathrm{mg} / \mathrm{dl})\end{array}$} \\
\hline
\end{tabular}


Table 2.3. Association between increasing serum levels of sVCAM-1 and sICAM-1 and incident diabetes, by gender $\int$

\begin{tabular}{|c|c|c|c|c|}
\hline & \multicolumn{2}{|r|}{ Women } & \multicolumn{2}{|r|}{ Men } \\
\hline & $\begin{array}{c}\text { No. at risk } \\
\text { (diabetes cases) }\end{array}$ & $\begin{array}{l}\text { Multivariable-adjusted hazards } \\
\text { ratio }(95 \% \text { confidence interval })\end{array}$ & $\begin{array}{l}\text { No. at risk } \\
\text { (diabetes } \\
\text { cases) }\end{array}$ & $\begin{array}{l}\text { Multivariable-adjusted hazards ratio } \\
(95 \% \text { confidence interval })\end{array}$ \\
\hline \multicolumn{5}{|c|}{$\begin{array}{l}\text { Serum sVCAM-1 tertiles* } \\
(\mathrm{n}=1119)\end{array}$} \\
\hline Tertile 1 & $208(23)$ & 1 (referent) & $162(19)$ & 1 (referent) \\
\hline Tertile 2 & $214(26)$ & $1.06(0.58,1.93)$ & $154(34)$ & $2.17,(1.16,4.05)$ \\
\hline Tertile 3 & $225(24)$ & $0.90(0.46,1.77)$ & $156(30)$ & $2.29(1.18,4.44)$ \\
\hline p-trend & & 0.7413 & & 0.0157 \\
\hline \multicolumn{5}{|c|}{$\begin{array}{l}\text { Serum sICAM-1 tertiles } \dagger \\
(\mathrm{n}=1119)\end{array}$} \\
\hline Tertile 1 & $203(22)$ & 1 (referent) & $167(21)$ & 1 (referent) \\
\hline Tertile 2 & $219(21)$ & $0.80(0.43,1.52)$ & $149(27)$ & $1.51(0.82,2.77)$ \\
\hline Tertile 3 & $225(30)$ & $1.26(0.69,2.31)$ & $156(35)$ & $1.88(1.01,3.48)$ \\
\hline p-trend & & 0.4086 & & 0.0451 \\
\hline
\end{tabular}

*sVCAM-1 Tertiles :Tertile $1(\leq 682.2 \mathrm{ng} / \mathrm{ml})$ Tertile $2(682.3-826.2 \mathrm{ng} / \mathrm{ml})$ Tertile $3(\geq 826.2 \mathrm{ng} / \mathrm{ml})$

†sICAM-1 Tertiles : Tertile $1(\leq 247.5 \mathrm{ng} / \mathrm{ml})$ Tertile $2(247.6-302.2 \mathrm{ng} / \mathrm{ml})$ Tertile $3(\geq 302.2 \mathrm{ng} / \mathrm{ml})$

†Adjusted for age (years), education categories (<high school, high school, > high school), smoking (never, former, current), alcohol intake (never, former, current), body mass index $\left(\mathrm{kg} / \mathrm{m}^{2}\right)$, hypertension (absent, present) and serum cholesterol (\%), high sensitivity C reactive protein (mg/dl)

$\int \mathrm{P}$-interaction for gender and sVCAM-1 $=0.1168$

$\int \mathrm{P}-$ interaction for gender and sICAM-1 $=0.2164$ 
Table 2.4. Association between increasing serum levels of sVCAM-1 and sICAM-1 and incident diabetes by hypertension status $\int$

\begin{tabular}{|c|c|c|c|c|}
\hline & \multicolumn{2}{|r|}{ No Hypertension } & \multicolumn{2}{|r|}{ Hypertension } \\
\hline & $\begin{array}{c}\text { No. at risk } \\
\text { (diabetes cases) }\end{array}$ & $\begin{array}{l}\text { Multivariable-adjusted hazards } \\
\text { ratio ( } 95 \% \text { confidence interval) } \$\end{array}$ & $\begin{array}{c}\text { No. at risk } \\
\text { (diabetes cases) }\end{array}$ & $\begin{array}{l}\text { Multivariable-adjusted hazards } \\
\text { ratio }(95 \% \text { confidence interval }) \ddagger\end{array}$ \\
\hline \multicolumn{5}{|l|}{$\begin{array}{l}\text { Serum sVCAM-1 tertiles* } \\
(\mathrm{n}=1119)\end{array}$} \\
\hline Tertile 1 & $225(25)$ & 1 (referent) & $145(17)$ & 1 (referent) \\
\hline Tertile 2 & $226(27)$ & $1.08(0.61,1.93)$ & $142(33)$ & $2.51(1.32,4.76)$ \\
\hline Tertile 3 & $183(21)$ & $1.07(0.56,2.05)$ & $198(33)$ & $2.03(1.03,3.99)$ \\
\hline $\mathrm{p}$-trend & & 0.8262 & & 0.0556 \\
\hline \multicolumn{5}{|l|}{$\begin{array}{l}\text { Serum sICAM-1 tertiles } \dagger \\
(\mathrm{n}=1119)\end{array}$} \\
\hline Tertile 1 & $225(24)$ & 1 (referent) & 145 (19) & 1 (referent) \\
\hline Tertile 2 & $192(21)$ & $1.12(0.60,2.09)$ & $176(27)$ & $1.17(0.63,2.18)$ \\
\hline Tertile 3 & $217(28)$ & $1.08(0.58,2.01)$ & $164(37)$ & $2.04(1.11,3.76)$ \\
\hline $\mathrm{p}$-trend & & 0.8022 & & 0.0186 \\
\hline \multicolumn{5}{|c|}{ 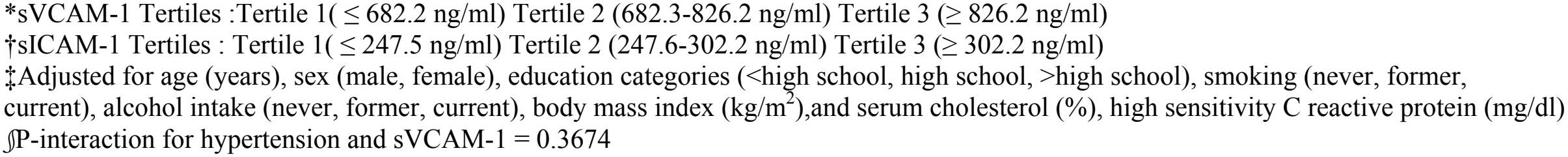 } \\
\hline
\end{tabular}




\section{Chapter 3}

Manuscript 2: The Association of Markers of Endothelial Dysfunction and Incident Hypertension 


\subsection{INTRODUCTION}

Endothelial dysfunction is a key pathophysiological process involved in the development of cardiovascular disease.$^{76}$ Endothelial dysfunction has also been shown to precede the development of hypertension in animal models ${ }^{112,113}$. However the relationship between markers of endothelial dysfunction and hypertension development in humans is not clear. Soluble adhesion molecules, including serum levels of vascular cell adhesion molecule-1 (sVCAM-1) and intercellular adhesion molecule -1 (sICAM-1) have been used as reliable markers of endothelial dysfunction in epidemiological studies. ${ }^{114}$ In humans, markers of endothelial dysfunction have been associated with hypertension in pregnant women,${ }^{85}$ subjects with type 1 diabetes ${ }^{86}$ and those with obstructive sleep apnea-hypopnea syndrome. ${ }^{87}$ Also, in the general population, Zhang et $\mathrm{al}^{88}$ showed that there was a positive association between SICAM-1 and Eselectin levels and hypertension in a cross-sectional survey from China. However, to our knowledge, no epidemiological study has examined the relationship between soluble adhesion molecules and the longitudinal risk of developing hypertension in the general population. In this context, we examined the relation between serum levels of SVCAM-1 and SICAM- 1 and the cumulative incidence of hypertension over a period of 10 years in a population-based sample of adults from Wisconsin. 


\subsection{METHODS}

\subsubsection{Population}

The current study is based on data from the Beaver Dam Eye study, a population-based cohort study in Wisconsin. The methods used to identify and describe the Beaver Dam population have appeared in previous reports. ${ }^{115,116}$ In brief, a private census of the population of Beaver Dam, Wisconsin, was performed from September 1987 to May 1988 to identify all residents in the city or township of Beaver Dam who were 43-84 years of age. Of the 5,924 eligible individuals (98\% Caucasians), 4,926 (83.1\%) participated in the baseline examination between March 1, 1988 and September 14, 1990. Comparisons between participants and nonparticipants at the time of the baseline examination have appeared elsewhere. ${ }^{116}$

The baseline examination was followed by a four follow-up examinations 5 year apart. We limit our analyses to the first 10-years of follow-up. Written informed consent was obtained from each subject at each examination. The study was approved by the Human Subjects Committee of the University of Wisconsin School of Medicine and Public Health, Madison, WI. Levels of sVCAM-1 and sICAM-1 were measured in stored frozen serum on a random sample of the baseline cohort $(\mathrm{n}=1793)$ as part of an ancillary study examining risk factors for kidney disease.

For the longitudinal analyses with sVCAM-1 and sICAM-1 as the main exposure, out of 1793 individuals with available sVCAM-1 and sICAM-1 measurements who participated in the baseline and at least the 5- or 10-year follow-up examination, we excluded subjects with prevalent hypertension at baseline $(\mathrm{n}=884)$ and missing information on covariates included in the multivariable model $(\mathrm{n}=28)$. This resulted in 881 participants without hypertension with 
complete biomarker and covariate information at baseline who were to form the at-risk population.

\subsubsection{Exposure ascertainment}

The baseline and follow-up examinations included measurement of weight, height, systolic and diastolic blood pressure by trained observers and administering a standardized questionnaire that collected information regarding participants' demographic characteristics, details regarding cigarette smoking, alcohol intake, medical histories and medications taken, including physician-diagnosed diabetes, hypertension or cardiovascular disease (CVD). Nonfasting blood specimens were obtained for measurement of plasma glucose, glycosylated hemoglobin, serum total cholesterol and high density lipoprotein (HDL) cholesterol.

Age was defined as the participants' age at the time of the baseline examination.

Education was categorized as less than high school, high school, or beyond high school. Body mass index (BMI) was defined as participants' weight in kilograms divided by their height in meters squared. Diabetes mellitus was defined by use of using insulin, oral hypoglycemic agents, or were newly classified as having diabetes based on the glycosylated hemoglobin value

that was $\geq 6.5 \%$, consistent with recent American Diabetes Association guidelines. ${ }^{104}$ Chronic kidney disease was defined as having chronic kidney disease were defined as having an estimated glomerular filtration rate (GFR) of $<60 \mathrm{ml} / \mathrm{min}$ per $\mathrm{m}^{2}$, estimated from serum creatinine employing the re-expressed Modification of Diet in Renal Diseases (MDRD) study equation. ${ }^{117}$

At cohort examinations, blood samples were collected in three 10-mL liquid EDTA blood tubes, placed on ice packs, stored in Styrofoam containers until they were centrifuged and 
aliquoted for storage in freezers at $-80^{\circ} \mathrm{C}$ until the time of laboratory analysis. Quality control samples were routinely frozen with study participant samples. Baseline frozen plasma samples were randomly ordered (to further reduce systematic bias and inter assay variation) and thawed and batch-analyzed for markers of endothelial function (sVCAM-1, sICAM-1).

sVCAM-1 (soluble vascular cell adhesion molecule-1) was measured in plasma or serum using the quantitative sandwich enzyme technique of the enzyme-linked immunosorbent assay (ELISA) Parameter kit from R \& D Systems (Minneapolis, MN). The intensity of the color was measured on a SpectraMax spectrophotometer (Molecular Devices, Sunnyvale, California). The inter-assay CV range was $8.9-10.2 \%$ for the plasma assay.

sICAM-1 (soluble intercellular adhesion molecule-1) was measured in plasma using the quantitative sandwich enzyme technique of the enzyme-linked immunosorbent assay (ELISA) Parameter kit from R \& D Systems (Minneapolis, MN). The intensity of the color was measured on a SpectraMax spectrophotometer (Molecular Devices, Sunnyvale, California). The interassay CV range was $6.0-10.1 \%$ for the plasma assay.

\subsubsection{Outcome of interest: Hypertension}

Hypertension was defined as having a systolic blood pressure of $140 \mathrm{mmHg}$ or higher, and/or a diastolic blood pressure $90 \mathrm{mmHg}$ or higher, and/or the combination of self-reported hypertension diagnosis by a physician and use of antihypertensive medications. Incident hypertension was defined as having newly diagnosed hypertension at the 5-, or 10 year follow-up examination among subjects free of hypertension at baseline. 


\subsubsection{Statistical methods}

We examined the association between serum levels of sVCAM-1 and sICAM-1 and 10year incident hypertension. We categorized sVCAM-1 and sICAM-1 into quartiles for the main analysis. We also analyzed these markers as continuous variables, as per standard deviation increase. We used chi-square test and analysis of variance to compare the relationship of selected baseline characteristics by increasing categories of sVCAM- 1 and sICAM-1. We used multivariable Cox proportional hazard regression models to determine the hazard ratio (HR) and 95 percent confidence interval (CI) of 10-year incident hypertension, controlling simultaneously for potential confounders, including age, gender (female, male), education $(<$ high school, high school, >high school), smoking (never, former, current), alcohol intake (never, former, current), BMI $\left(\mathrm{kg} / \mathrm{m}^{2}\right)$, diabetes (absent, present), serum total cholesterol ( $\mathrm{mg} / \mathrm{dL}$ ), chronic kidney disease (absent, present) and self-reported history of cardiovascular disease (absent, present). To further validate the associations between categories of SVCAM-1 and SICAM-1, we stratified by gender (men, women), current smoking status (yes, no), obesity status (absent, present), diabetes status (absent, present), glycosylated hemoglobin levels (\%), and chronic kidney disease status (absent, present). All analyses were performed in SAS version 9.2 (SAS Institute, Cary, NC). 


\subsection{RESULTS}

Among 881 study participants included in the current analysis, there were 390 men and 491 women. Overall, 257 subjects (29\%) developed hypertension over an average 10-year follow-up period, including 106 men and 151 women.

Table 3.1 presents the characteristics of the study population by increasing serum sVCAM-1 levels at baseline. Those who had higher serum sVCAM-1 levels were more likely to be older, never or former smokers. They were also likely to have higher systolic blood pressure but lower diastolic blood.

Table 3.2 presents the association between increasing categories of serum sVCAM-1, sICAM-1 and 10-year incident hypertension. The multivariable model for sVCAM-1 showed a positive association between increasing serum sVCAM-1 levels and incident hypertension. The model evaluating the trend was statistically significant $(\mathrm{p}$-trend $=0.0005)$. However, the multivariable model for sICAM-1 showed no positive association between increasing serum sICAM-1 levels and incident hypertension ( $\mathrm{p}$-trend $=0.3203$ ).

Table 3.3 presents the association between increasing categories of serum sVCAM-1 and sICAM-1 and 10-year incident hypertension by gender. For sVCAM-1, the multivariable model showed a positive association with incident hypertension in women as well as men. However, for sVCAM-1 there was no association with incident hypertension in either sex.

Table 3.4 presents the associations between increasing categories of serum sVCAM-1, sICAM-1 and incident hypertension, stratified by smoking, obesity, diabetes, and chronic kidney disease status. Here also, generally in agreement with Table 2, the multivariable model for sVCAM-1 showed a positive association with incident hypertension, but not for sICAM-1. Formal statistical tests for interaction were not significant (all p-interactions $>0.10$ ). 
In a supplementary analysis when we adjusted for additional factors that may serve as confounders, including the use of medications that may affect endothelial function such as statins (current user, nonuser) and aspirin (current user, nonuser), and novel biomarkers such as high sensitivity C-reactive protein level $(\mathrm{mg} / \mathrm{dL})$ and serum uric acid $(\mathrm{mg} / \mathrm{dL})$, the association between increasing SVCAM-1 and sICAM-1 and hypertension remained materially the same. For sVCAM1, compared to quartile 1 (referent), the multivariable HR (95\% CI) of incident hypertension was $1.39(0.92,2.10)$ in quartile $2,1.75(1.16,2.64)$ in quartile 3 , and 1.98 (1.39, 2.82); $\mathrm{p}$-trend $=0.0004$. Also, for sICAM-1, compared to quartile 1 (referent), the multivariable HR $(95 \% \mathrm{CI})$ of incident hypertension was $1.29(0.81,2.05)$ in quartile $2,1.09(0.74,1.61)$ in quartile 3 , and $1.18(0.80,1.74) ; \mathrm{p}$-trend $=0.432$. 


\subsection{DISCUSSION}

In a population-based sample of adults from Wisconsin, increasing serum levels of sVCAM-1 were found to be positively associated with incident hypertension, independent of smoking, alcohol intake, education, hypertension, body mass index, serum total cholesterol, and chronic kidney disease. Serum sICAM-1, in contrast, was not found to be associated with hypertension.

Serum levels of sVCAM-1 and sICAM-1 have been used previously in epidemiological research as reliable markers of endothelial dysfunction. ${ }^{114}$ Previous studies have reported that markers of endothelial dysfunction are associated with increased risk of developing cardiovascular disease. ${ }^{76}$ As hypertension is a strong risk factor for cardiovascular disease, we examined if endothelial dysfunction markers are also related to hypertension. Subsequently we found that serum SVCAM-1 was positively related to incident hypertension in both men and women.

The observed association between endothelial dysfunction and hypertension may be due to endothelial damage and dysfunction impairing the vasodilator mechanism of endotheliumderived relaxing factor (nitric oxide), subsequently causing elevated blood pressure. ${ }^{63}$ Endothelial dysfunction may also be related to other mechanisms involved in the development of hypertension, including insulin resistance ${ }^{64}$, systemic inflammation ${ }^{65}$ and hyperuricemia. ${ }^{66}$ However, in the current study, in a supplementary analysis, the association between sVCAM-1 and hypertension was found to be attenuated, but still present, after adjustment for markers of inflammation and uric acid, suggesting that these mechanisms may only partially explain the observed association. 
Our findings are consistent with one previous prospective epidemiological study examining the relationship between biomarkers of endothelial dysfunction and hypertension in those with type 1 diabetes ${ }^{86}$ and another cross-sectional study conducted in China. ${ }^{88}$ However, another epidemiological study, the Multiethnic Study of Atherosclerosis (MESA), found no association of endothelial dysfunction measured by brachial artery flow mediated-dilation with incident hypertension. ${ }^{118}$ The reason for this discrepancy in findings with our study is not clear. It is possible that serum levels of cellular adhesion molecules and brachial artery flow-mediated dilatation may be measuring different aspects of endothelial function. For example, physiological monitoring of flow-mediated dilatation is considered to be more dynamic and probably acutely influenced by a subject's recent dietary ${ }^{119}$ or recent exposure to environmental pollution. ${ }^{120}$ Also, studies in animals ${ }^{121}$ and humans ${ }^{122}$ have suggested that under different conditions (e.g. prolonged hyperemic stimulus vs. transient increases in blood flow), flow mediated dilatation may occur by mechanisms that are independent of endothelial nitric oxide production. ${ }^{123}$ Another possibility is that there are potential racial/ethnic differences in the endothelial dysfunctionhypertension association as our study consisted of $99 \%$ whites whereas the MESA was a multiethnic sample.

The reasons for the differing results that we observed for sVCAM-1 and sICAM-1 with incident hypertension are not clear. It is possible that even though these adhesion molecules are similar in structure and are members of the cytokine-inducible immunoglobulin gene superfamily ${ }^{124}$. they have different functions. This notion is also supported by recent animal studies that have shown that VCAM-1, and not ICAM-1, plays a major role in the initiation of atherosclerosis $^{125}$, and others that have reported differential expression patterns for these adhesion molecules with VCAM-1 being expressed more in atherosclerotic lesions whereas 
ICAM-1 expression extending into uninvolved aorta and lesion protected regions ${ }^{126}$. However, there is a need for more human studies to validate or refute our findings.

Strengths of this study include it being a large population-based cohort, high participation rate, use of standardized protocols for exposure and outcome measurement and the availability of specific markers of endothelial dysfunction, including sVCAM-1 and sICAM-1 levels. Our results possess strong internal validity as we adjusted for known confounders and also the results remained relatively consistent when we performed stratified analysis by confounders. Study limitations include selective survival, the potential variability of endothelial dysfunction tests and the limited generalizability of our study to other populations as this sample is a $99 \%$ white.

In conclusion, in a population-based sample of Wisconsin adults, we found that increasing serum levels of sVCAM-1 were positively associated with 10-year incident hypertension, independent of age smoking status, alcohol intake, education, hypertension, BMI, total cholesterol levels, chronic kidney disease and history of cardiovascular disease. In contrast, serum sICAM-1 was not found to be associated with hypertension. 


\subsection{TABLES}

Table 3.1. Baseline characteristics by soluble vascular cell adhesion molecule 1 (sVCAM -1) Quartile

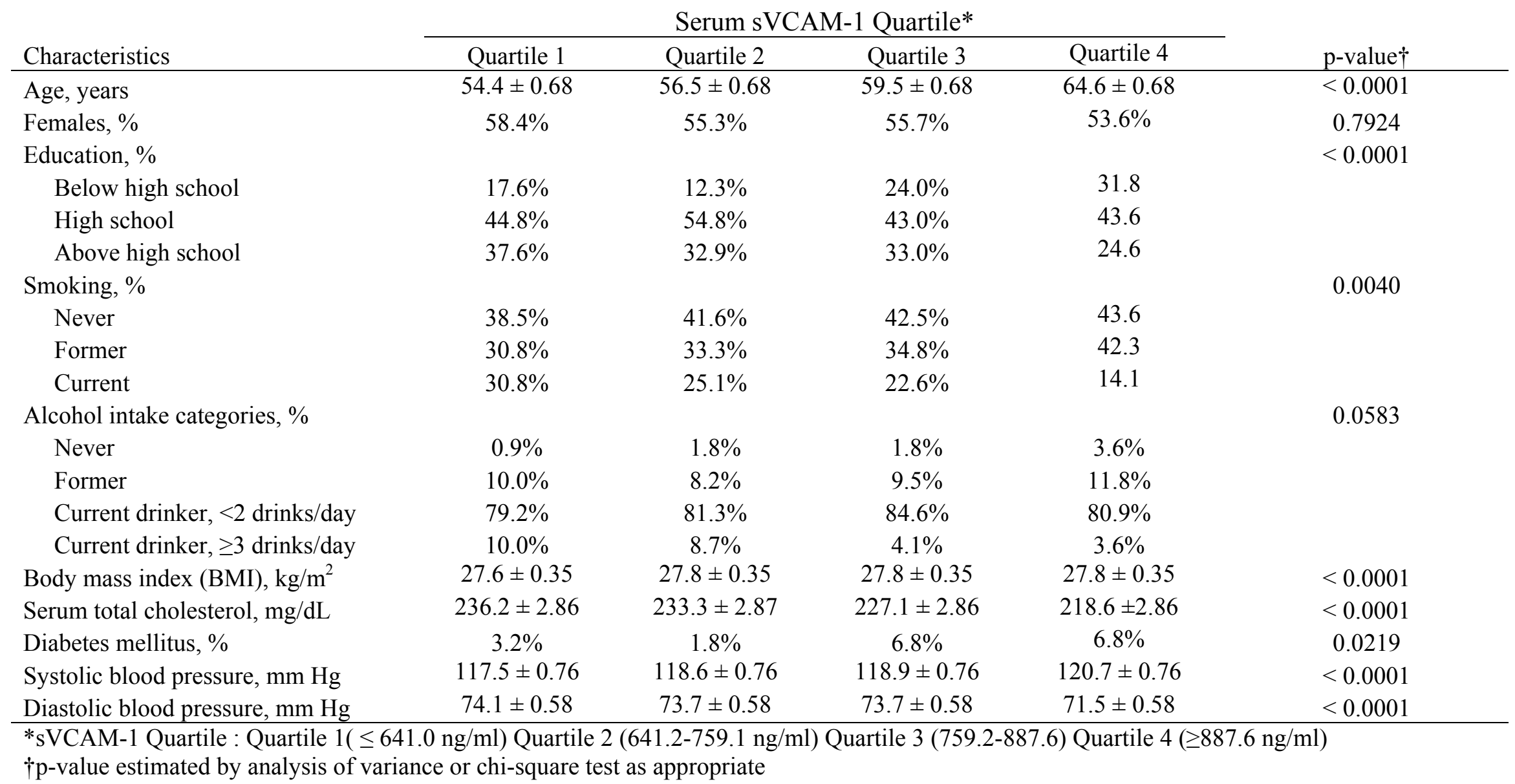


Table 3.2. Association between increasing serum levels of sVCAM-1 and sICAM-1 and incident hypertension

\begin{tabular}{lcccc}
\hline Serum sVCAM-1 quartiles* & $\begin{array}{c}\text { No. at risk } \\
(\mathrm{n}=881)\end{array}$ & $\begin{array}{c}\text { Incident } \\
\text { Hypertension } \\
(\mathrm{n}=257)\end{array}$ & $\begin{array}{c}\text { Unadjusted hazards ratio }(95 \% \\
\text { confidence interval) }\end{array}$ & $\begin{array}{c}\text { Multivariable-adjusted } \\
\text { hazards ratio (95\% confidence interval) } !\end{array}$ \\
\hline Quartile 1 & 221 & 46 & 1 (referent category) & 1 (referent category) \\
Quartile 2 & 219 & 65 & $1.44(0.95,2.18)$ & $1.47(0.96,2.26)$ \\
Quartile 3 & 221 & 72 & $1.81(1.20,2.74)$ & $1.78(1.17,2.73)$ \\
Quartile 4 & 220 & 74 & $2.48(1.63,3.76)$ & $2.14(1.42,3.23)$ \\
p-trend & & & $<0.0001$ & 0.0003
\end{tabular}

Serum sICAM-1 quartiles $\dagger$

$\begin{array}{rcccc}\text { Quartile 1 } & 221 & 56 & 1 \text { (referent category) } & 1 \text { (referent category) } \\ \text { Quartile 2 } & 219 & 66 & 1.33(0.89,1.98) & 1.33(0.88,2.00) \\ \text { Quartile 3 } & 221 & 69 & 1.36(0.91,2.01) & 1.15(0.76,1.74) \\ \text { Quartile 4 } & 220 & 66 & 1.58(1.05,2.36) & 1.26(0.85,2.09) \\ \text { p-trend } & & & 0.0322 & 0.3603\end{array}$

*sVCAM-1 Quartiles: Quartile 1 (0-641.0 (ng/ml)), Quartile 2 (641.1-759.1 ng/ml), Quartile 3 (759.2-887.6), Quartile 4 ( > 887.6 ng/ml) $\dagger$ sICAM-1 Quartiles: Quartile 1 (0-233.9 (ng/ml)), Quartile 2 (234.0-277.8 ng/ml), Quartile $3(277.9-334.2)$, Quartile $4(>334.2 \mathrm{ng} / \mathrm{ml})$ $\$$ Adjusted for age (years), gender (male, female), education categories ( $<$ high school, high school, $>$ high school), smoking (never, former, current), alcohol intake (never, former, current), body mass index $\left(\mathrm{kg} / \mathrm{m}^{2}\right)$, diabetes (absent, present), glycosylated hemoglobin (\%), chronic kidney disease (absent, present), history of cardiovascular disease (absent, present) and serum cholesterol (mg/dL) 
Table 3.3. Association between increasing serum levels of sVCAM-1 and sICAM-1 and incident hypertension stratified by gender.

\begin{tabular}{|c|c|c|}
\hline & $\operatorname{Men}(n=390)$ & Women $(n=491)$ \\
\hline Serum sVCAM-1 quartiles* & $\begin{array}{c}\text { Multivariable-adjusted } \\
\text { hazards ratio }(95 \% \text { confidence interval })\end{array}$ & $\begin{array}{c}\text { Multivariable-adjusted } \\
\text { hazards ratio }(95 \% \text { confidence interval })\end{array}$ \\
\hline Quartile 1 & 1 (referent category) & 1 (referent category) \\
\hline Quartile 2 & $0.97(0.48,1.94)$ & $1.95(1.11,3.40)$ \\
\hline Quartile 3 & $1.69(0.89,3.21)$ & $1.88(1.05,3.36)$ \\
\hline Quartile 4 & $1.81(1.01,3.24)$ & $2.86(1.57,5.22)$ \\
\hline p-trend & 0.0440 & 0.0013 \\
\hline \multicolumn{3}{|l|}{ Serum sICAM-1 quartiles $\dagger$} \\
\hline Quartile 1 & 1 (referent category) & 1 (referent category) \\
\hline Quartile 2 & $1.04(0.55,1.97)$ & $1.59(0.92,2.77)$ \\
\hline Quartile 3 & $0.84(0.43,1.65)$ & $1.39(0.83,2.33)$ \\
\hline Quartile 4 & $1.35(0.71,2.57)$ & $1.28(0.71,2.31)$ \\
\hline p-trend & 0.5100 & 0.4798 \\
\hline
\end{tabular}

* sVCAM-1: soluable vascular cell adhesion molecule-1; sVCAM Quartiles Quartile 1 (0-641.0 (ng/ml)), Quartile 2 (641.1-759.1 ng/ml), Quartile 3 (759.2-887.6), Quartile 4 ( > 887.6 ng/ml)

†sICAM-1: soluable intercellular adhesión molecule-1; sICAM Quartiles: Quartile 1 (0-233.9 (ng/ml)), Quartile 2 (234.0-277.8 ng/ml), Quartile 3 (277.9-334.2), Quartile 4 ( > $334.2 \mathrm{ng} / \mathrm{ml})$

\$Adjusted for age (years), education categories (<high school, high school, $>$ high school), smoking (never, former, current), alcohol intake (never, former, current), body mass index $\left(\mathrm{kg} / \mathrm{m}^{2}\right)$, diabetes (absent, present), glycosylated hemoglobin (\%), chronic kidney disease (absent, present), history of cardiovascular disease (absent, present) and serum cholesterol (mg/dL) 
Table 3.4. The association by sVCAM-1 and sICAM-1 and incident hypertension in selected subgroups

\section{sVCAM-1}

The multivariable-adjusted hazards ratio $\uparrow$ (95\% confidence interval) for

\section{Categories} No. at risk one standard deviation increase

\section{sICAM-1}

The multivariable-adjusted hazards ratio $\uparrow$ (95\% confidence interval) for one standard deviation increase $\ddagger$

\section{Current Smoker}

$$
\text { No }
$$

677

$1.16(0.96,1.40)$

$0.98(0.80,1.19)$

Yes

$1.26(0.78,2.04)$

$1.21(0.88,1.66)$

Obesity

\section{Absent}

Present

$1.17(0.93,1.48)$

$1.23(0.81,1.87)$

$1.05(0.88,1.25)$

$0.88(0.63,1.23)$

Diabetes

$\begin{array}{ll}\text { Absent } & 840 \\ \text { Present } & 41\end{array}$

$1.27(1.01,1.57)$

$1.05(0.90,1.23)$

Present

$1.16(0.67,2.01)$

$0.28(0.03,2.46)$

Chronic kidney disease

$\begin{array}{lccr}\text { Absent } & 783 & 1.16(0.95,1.41) & 1.12(0.93,1.35) \\ \text { Present } & 98 & 1.26(0.70,2.27) & 0.55(0.27,1.12)\end{array}$

*All p-interactions $>0.10$

$\dagger$ Adjusted for age (years), gender (male, female), education categories (<high school, high school, $>$ high school), smoking (never, former, current), alcohol intake (never, former, current), body mass index ( $\left.\mathrm{kg} / \mathrm{m}^{2}\right)$, diabetes (absent, present), glycosylated hemoglobin (\%), chronic kidney disease (absent, present), history of cardiovascular disease (absent, present) and serum cholesterol (mg/dL); not adjusted for stratifying variable.

$\ddagger$ one standard deviation sVCAM-1 = 323.0 units; one standard deviation of sICAM-1 = 99.2 units 


\section{Chapter 4}

Manuscript 3: The Association of Markers of Endothelial Dysfunction and Incident Chronic Kidney Disease 


\subsection{INTRODUCTION}

Chronic kidney disease (CKD) is recognized as a risk factor for cardiovascular disease ${ }^{35}$, and it also independently predicts cardiovascular mortality. ${ }^{36}$ Endothelial dysfunction is one of the key pathophysiologic processes involved in the development of atherosclerotic vascular disease. ${ }^{127}$ Binding and recruitment of circulating leukocytes to the vascular endothelium and further migration into the subendothelial spaces are mediated through a diverse family of cellular adhesion molecules that are expressed on the surface of vascular endothelial cells, including sVCAM-1 and sICAM-1. ${ }^{128}$ Circulating levels of these adhesion molecules have been used as markers of endothelial function in epidemiologic studies. ${ }^{129}$ Studies have shown that markers of endothelial dysfunction, including sVCAM-1 and sICAM-1 are related to risk of developing diabetes mellitus ${ }^{130}$ and hypertension, ${ }^{131}$ two of the well known risk factors of CKD.

Previous cross-sectional studies have shown that markers of endothelial function are related to kidney disease ${ }^{132-134}$. However, since endothelial function markers are also elevated secondary to CKD and dialysis, ${ }^{89,90,135}$ studies with longitudinal follow-up are required to examine whether endothelial dysfunction precedes CKD onset. Therefore, we examined biomarkers of endothelial dysfunction, including sVCAM-1 and sICAM-1 in relationship to the 15-year cumulative incidence of CKD in a population-based sample of adults from Wisconsin. 


\subsection{METHODS}

\subsubsection{Population}

The current study is based on data from the Beaver Dam Eye study, a population-based cohort study in Wisconsin originally aimed at studying age-related eye diseases. The methods used to identify and describe the Beaver Dam population have appeared in previous reports. ${ }^{115,116}$ In brief, a private census of the population of Beaver Dam, Wisconsin, was performed from September 1987 to May 1988 to identify all residents in the city or township of Beaver Dam who were 43-84 years of age. Of the 5,924 eligible individuals (98\% Caucasians), 4,926 (83.1\%) participated in the baseline examination between March 1, 1988 and September 14, 1990. Comparisons between participants and nonparticipants at the time of the baseline examination have appeared elsewhere ${ }^{116}$.

The baseline examination was followed by a 5-year follow-up examination from 1993 to 1995, a 10-year follow-up examination from 1998-2000, and a 15-year follow-up examination from 2003-2005. Written informed consent was obtained from each subject at each examination. The study was approved by the Human Subjects Committee of the University of Wisconsin School of Medicine and Public Health, Madison, WI. Serum levels of sVCAM-1 and sICAM-1 were measured on a random sample of the baseline cohort $(\mathrm{n}=1793)$ as part of an ancillary study examining risk factors for kidney disease.

For the longitudinal analyses with SVCAM-1 and sICAM-1 as the main exposure, out of 1793 individuals with available sVCAM-1 and sICAM-1 measurements who participated in the baseline and at least one follow-up examination, we excluded subjects with CKD at baseline $(\mathrm{n}=141)$, missing information on covariates included in the multivariable model $(\mathrm{n}=343)$ and 
those who had a history of cardiovascular disease $(n=264)$. This resulted in $n=1045$ CKD-free individuals with biomarker complete covariate information to form the at-risk study population.

\subsubsection{Exposure ascertainment}

The baseline and follow-up examinations included measurement of weight, height, systolic and diastolic blood pressure by trained observers and administering standardized questionnaire that collected information regarding participants' demographic characteristics, details regarding cigarette smoking, alcohol intake, medical histories and medications taken, including physician-diagnosed diabetes, hypertension or cardiovascular disease (CVD). Nonfasting blood specimens were obtained for measurement of plasma glucose, glycosylated hemoglobin, serum total cholesterol and high density lipoprotein (HDL) cholesterol.

Age was defined as the participants' age at the time of the baseline examination.

Education was categorized as less than high school, high school, or beyond high school. Body mass index (BMI) was defined as participants' weight in kilograms divided by their height in meters squared. Hypertension was defined as a systolic blood pressure of $140 \mathrm{mmHg}$ or higher, and/or a diastolic blood pressure $90 \mathrm{mmHg}$ or higher, and/or the combination of self-reported hypertension diagnosis by a physician and use of antihypertensive medications.

At cohort examinations, blood samples were collected in three 10-mL liquid EDTA blood tubes, placed on ice packs, stored in Styrofoam containers until they were centrifuged and aliquoted for storage in freezers at $-80^{\circ} \mathrm{C}$ until the time of laboratory analysis. Quality control samples were routinely frozen with study participant samples. Baseline frozen plasma samples were randomly ordered (to further reduce systematic bias and inter assay variation) and thawed and batch-analyzed for markers of endothelial function (sVCAM-1, sICAM-1). 
sVCAM-1 (soluble vascular cell adhesion molecule-1) was measured in serum using the quantitative sandwich enzyme technique of the enzyme-linked immunosorbent assay (ELISA) Parameter kit from R \& D Systems (Minneapolis, MN). The intensity of the color was measured on a SpectraMax spectrophotometer (Molecular Devices, Sunnyvale, California). The interassay coefficient of variability (CV) range was $8.9-10.2 \%$.

sICAM-1 (soluble intercellular adhesion molecule-1) was measured in serum using the quantitative sandwich enzyme technique of the enzyme-linked immunosorbent assay (ELISA) Parameter kit from R \& D Systems (Minneapolis, MN). The intensity of the color was measured on a SpectraMax spectrophotometer (Molecular Devices, Sunnyvale, California). The interassay $\mathrm{CV}$ range was $6.0-10.1 \%$.

\subsubsection{Outcome of Interest: Chronic Kidney Disease}

Chronic kidney disease was defined as having an estimated glomerular filtration rate (eGFR) of $<60 \mathrm{ml} / \mathrm{min}$ per $\mathrm{m}^{2}$, using the re-expressed Modification of Diet in Renal Diseases (MDRD) study equation ${ }^{117}$ to estimate GFR for the main analysis and the CKD-EPI equation in a supplementary analysis to verify the results. ${ }^{136}$ Incident CKD was defined as having newly diagnosed CKD at the 5-, 10-, or 15-year follow-up examination among subjects free of CKD at baseline.

\subsubsection{Statistical methods}

We were interested in the association between markers of endothelial dysfunction (sVCAM-1 and sICAM-1 levels) and incident CKD. We categorized sVCAM-1 and sICAM-1 
into quartiles for the main analysis. We also analyzed these markers as continuous variables after logarithmic transformation due to their skewed distribution.

We used chi-square test and analysis of variance to compare the relationship of selected baseline characteristics to incident CKD status. We used multivariable Cox proportional hazard regression models to determine the hazard ratio (HR) and 95 percent confidence interval (CI) of 15-year incident CKD, controlling simultaneously for potential confounders. We used two nested Cox proportional hazard regression models: an unadjusted model and a multivariable-adjusted model, additionally adjusted for age, gender (male, female), education ( $<$ high school, high school, >high school), smoking (never, former, current), alcohol intake (never, former, current), BMI $\left(\mathrm{kg} / \mathrm{m}^{2}\right)$, hypertension (absent, present), diabetes (absent, present) mean arterial blood pressure $(\mathrm{mm} \mathrm{Hg})$, and serum total cholesterol $(\mathrm{mg} / \mathrm{dL})$. To further validate the associations between increasing categories of sVCAM-1 and sICAM-1, we stratified by gender (men, women) and hypertension status (hypertension, no hypertension). 


\subsection{RESULTS}

Among 1045 Wisconsin adults $\geq 43$ years of age included in the current analysis, there were 466 men and 579 women. Overall, 269 subjects developed CKD, including 107 men and 162 women.

Table 4.1 presents the characteristics of the study population by chronic disease status. Those who had CKD were more likely to be older, female, have less than a high school education, be never smokers, current or former drinkers, have hypertension and have higher BMI, serum total cholesterol, and systolic and diastolic blood pressures.

Table 4.2 presents the associations between increasing categories of sVCAM-1, sICAM-1 and the 15-year cumulative incidence of CKD. The unadjusted-model for sVCAM-1 showed a positive association between increasing levels of sVCAM-1 and incident CKD. However, the multivariable-model for sVCAM-1 showed no association between increasing levels of sVCAM1 and incident CKD. Neither the unadjusted or multivariable models for sICAM-1 showed an association between increasing levels of sICAM-1 and incident CKD.

Table 4.3 presents the associations between increasing categories of sVCAM-1 and sICAM-1 and 15-year incident CKD by gender. For both men and women, the multivariable model for sVCAM-1 as well as sICAM-1 showed no association between increasing levels of these markers and incident CKD.

Table 4.4 presents the associations between increasing categories of sVCAM-1 and sICAM-1 and 15-year incident CKD by hypertension status. For both without and with hypertension, the multivariable model for sVCAM-1 as well as SICAM-1 showed no association between increasing levels of these markers and incident CKD. 
In a supplementary analysis, to examine the robustness of our findings, we analyzed the associations between increasing categories of sVCAM-1, sICAM-1 and 15-year incident CKD, employing the CKD-EPI equation instead of the MDRD equation; the results were found to be essentially similar with no association between these markers and incident CKD. For sVCAM-1, compared to quartile 1 (referent), the multivariable hazards ratio ( $95 \%$ confidence interval) of incident CKD was $0.77(0.46,1.28)$ in quartile $2,1.16(0.73,1.85)$ in quartile 3 , and $0.90(0.55$, 1.45) in quartile $4 ; \mathrm{p}$-trend $=0.9471$. For ICAM-1, compared to quartile 1 (referent), the multivariable hazards ratio $(95 \%$ confidence interval) of incident CKD was $1.15(0.71,1.86)$ in quartile $2,1.43(0.91,2.27)$ in quartile 3 , and $1.20(0.73,1.99)$ in quartile 4 ; $p$-trend $=0.3082$. 


\subsection{DISCUSSION}

In a population-based sample, after adjusting for lifestyle and medical risk factors, including gender, smoking status, alcohol intake, education, hypertension, body mass index and total cholesterol increasing levels of sVCAM-1 and sICAM-1 were not associated with incident CKD.

The vascular endothelium is involved in a variety of key processes important to human health and disease, including control of coagulation ${ }^{137}$, fibrinolysis ${ }^{137,138}$ vascular tone ${ }^{137,139}$ and growth $^{139}$, immune response ${ }^{137,139}$, and oxidative stress ${ }^{138,140}$. Endothelial dysfunction has been found to be closely related to insulin resistance ${ }^{141,142}$, elevated levels of inflammatory markers ${ }^{141,143}$, impaired function of lipoprotein lipase (contributing to high triglycerides and low HDL cholesterol levels) ${ }^{141,143}$, impaired nitric oxide release ${ }^{143}$ and endothelium dependent vasodilation $^{143}$. Endothelial dysfunction is also present in end-stage renal disesease ${ }^{134,144}$. Biomarkers of endothelial dysfunction, including circulating adhesion molecules VCAM-1 and ICAM-1 have been shown to be elevated even in less advanced stages of kidney disease ${ }^{132-134}$.

Several lines of recent evidence from animal studies support this hypothesis that biomarkers of endothelial dysfunction may predict CKD. Janssen et al. ${ }^{145}$ reported that ICAM-1 knockout mice developed significantly less severe experimentally induced nephrotoxic nephritis and glomerular crescents compared to control mice. Similarly, Kawasaki et al. showed that injection of antibodies against ICAM-1 prevented glomerular injury in experimental crescentic glomerular nephritis ${ }^{146}$. To our knowledge, this is the first prospective study in humans to examine the association between endothelial dysfunction and the development of renal disease using circulating levels of cellular adhesion molecules. Circulating levels of cellular adhesion 
molecules are specific and valid markers of endothelial dysfunction and have been used extensively in epidemiologic studies that utilized stored biospecimen ${ }^{130,131}$.

In the current study, we found that sVCAM-1 levels were initially moderately associated with CKD in the unadjusted model but after adjusting for lifestyle and medical risk factors, the association was no longer present. In contrast, sICAM-1 levels were not associated with CKD in the unadjusted as well as the multivariable-adjusted model. The reasons for the observed differential associations in CKD in the unadjusted model for sVCAM-1 and sICAM-1 are not clear. Analogous to the recently reported differential associations of these adhesion molecules in the brain microvasculature ${ }^{147}$ it is possible that VCAM-1 and ICAM-1 may have differential roles in leukocyte adhesion in renal microvessels also, with probably sVCAM-1 playing the major role of leukocyte recruitment in kidneys.

This study has high internal validity as we adjusted for the main confounders and performed stratified analyses by gender and hypertension status. Also, in contrast to Perticone, et $\mathrm{al}^{148}$ who reported an association between endothelial dysfunction markers and CKD among those with hypertension, the null association that we observed between markers of endothelial dysfunction and CKD was consistently present in those with and without hypertension.

Strengths of this study include its population-based sample, high participation rate, use of standardized protocols for exposure and outcome measurement and the availability of specific markers of endothelial dysfunction, including sVCAM-1 and sICAM-1 levels. Our results of no association between markers of endothelial dysfunction and incident CKD possess strong internal validity as we adjusted for known confounders and also the results remained relatively consistent when we performed stratified analysis by gender. A main study limitation is the 
generalizability of our study to other populations as this sample is a $99 \%$ white and is greater than or equal to 43 years of age.

In conclusion, in a population-based sample of adults from Wisconsin, increasing levels of sVCAM-1 and of sICAM-1 were not associated with incident CKD. Therefore, markers of endothelial dysfunction such as SVCAM-1 and sICAM-1 may not be independently associated with CKD. 


\subsection{TABLES}

Table 4.1. Baseline characteristics by chronic kidney disease (CKD) status

\begin{tabular}{|c|c|c|c|}
\hline \multirow[b]{2}{*}{ Characteristics } & \multicolumn{2}{|c|}{ CKD status } & \multirow[b]{2}{*}{ p-value $\dagger$} \\
\hline & No CKD & CKD & \\
\hline Age, years & $57.3 \pm 0.35$ & $61.7 \pm 0.59$ & $<0.0001$ \\
\hline Females, \% & $53.7 \%$ & $60.2 \%$ & 0.0652 \\
\hline Education, \% & & & 0.0005 \\
\hline Below high school & $16.8 \%$ & $27.1 \%$ & \\
\hline High school & $49.9 \%$ & $40.2 \%$ & \\
\hline Above high school & $33.4 \%$ & $32.7 \%$ & \\
\hline Smoking, \% & & & 0.0314 \\
\hline Never & $41.9 \%$ & $50.2 \%$ & \\
\hline Former & $36.7 \%$ & $28.6 \%$ & \\
\hline Current & $21.4 \%$ & $21.2 \%$ & \\
\hline Alcohol intake categories, $\%$ & & & 0.4570 \\
\hline Never & $3.1 \%$ & $2.6 \%$ & \\
\hline Former & $7.0 \%$ & $7.4 \%$ & \\
\hline Current drinker, $<2$ drinks/day & $80.2 \%$ & $83.3 \%$ & \\
\hline Current drinker, $\geq 3$ drinks/day & $9.8 \%$ & $6.7 \%$ & \\
\hline Body mass index (BMI), $\mathrm{kg} / \mathrm{m}^{2}$ & $28.6 \pm 0.20$ & $29.2 \pm 0.35$ & $<0.0001$ \\
\hline Serum total cholesterol, $\%$ & $229.1 \pm 1.19$ & $238.1 \pm 2.54$ & $<0.0001$ \\
\hline Hypertension, \% & $39.7 \%$ & $54.6 \%$ & \\
\hline Systolic blood pressure, $\mathrm{mm} \mathrm{Hg}$ & $128.2 \pm 0.67$ & $133.4 \pm 1.14$ & $<0.0001$ \\
\hline Diastolic blood pressure, $\mathrm{mm} \mathrm{Hg}$ & $78.27 \pm 0.37$ & $78.94 \pm 0.63$ & $<0.0001$ \\
\hline
\end{tabular}

$\dagger \mathrm{p}$-value estimated by analysis of variance or chi-square test as appropriate 
Table 4.2. Association between increasing serum levels of sVCAM-1 and sICAM-1 and incident CKD

\begin{tabular}{lcccc}
\hline $\begin{array}{l}\text { Serum sVCAM-1 quartiles* } \\
(\mathrm{n}=1045)\end{array}$ & No. at risk & $\begin{array}{c}\text { Cases } \\
(\mathrm{n}=269)\end{array}$ & $\begin{array}{c}\text { Unadjusted hazards ratio }(95 \% \\
\text { confidence interval) }\end{array}$ & $\begin{array}{c}\text { Multivariable-adjusted } \\
\text { hazards ratio (95\% confidence interval) } \neq\end{array}$ \\
\hline Quartile 1 & 260 & 62 & 1 (referent category) & 1 (referent category) \\
Quartile 2 & 262 & 52 & $0.87(0.60,1.25)$ & $0.82(0.55,1.23)$ \\
Quartile 3 & 262 & 75 & $1.29(0.92,1.81)$ & $1.15(0.79,1.67)$ \\
Quartile 4 & 261 & 80 & $1.53(1.10,2.13)$ & $1.14(0.77,1.68)$ \\
$\quad$ p-trend & & & 0.0021 & 0.2662 \\
Log-transformed sVCAM-1 & & $2.23(1.41,3.53)$ & $1.54(0.91,2.60)$ \\
& & & & 1 (referent category) \\
Serum sICAM-1 quartiles $\dagger$ & & & 1 (referent category) & $1.03(0.70,1.51)$ \\
(n=1045) & 262 & 60 & $1.16(0.82,1.64)$ & $1.05(0.72,1.54)$ \\
Quartile 1 & 260 & 69 & $1.32(0.94,1.86)$ & $1.04(0.69,1.55)$ \\
Quartile 2 & 73 & $1.18(0.83,1.67)$ & 0.8466 \\
Quartile 3 & 261 & 67 & 0.2707 & $1.33(0.79,2.25)$ \\
Quartile 4 & 262 & & $1.54(1.00,2.38)$ &
\end{tabular}

*sVCAM Quartiles: Quartile 1 (0-638.8 (ng/ml)), Quartile 2 (638.9-755.2 ng/ml), Quartile 3 (755.3-878.7), Quartile 4 (>878.7 ng/ml) $\dagger$ †ICAM-1: soluble intercellular adhesión molecule-1; sICAM Quartiles: Quartile 1 (0-234.2 (ng/ml)), Quartile 2 (234.3-278.4 ng/ml), Quartile 3 (278.5324.6), Quartile 4 ( > $342.6 \mathrm{ng} / \mathrm{ml}$ )

†Adjusted for age (years), education categories (<high school, high school, $>$ high school), smoking (never, former, current), alcohol intake (never, former, current), body mass index $\left(\mathrm{kg} / \mathrm{m}^{2}\right)$, hypertension (absent, present) and serum cholesterol (\%) 
Table 4.3. Association between increasing serum levels of sVCAM-1 and sICAM-1 and incident CKD stratified by gender

\begin{tabular}{|c|c|c|}
\hline & Men & Women \\
\hline Serum sVCAM-1 quartiles* $(n=1045)$ & $\begin{array}{c}\text { Multivariable-adjusted } \\
\text { hazards ratio }(95 \% \text { confidence interval }) \\
\end{array}$ & $\begin{array}{c}\text { Multivariable-adjusted } \\
\text { hazards ratio }(95 \% \text { confidence interval }) \ddagger\end{array}$ \\
\hline Quartile 1 & 1 (referent category) & 1 (referent category) \\
\hline Quartile 2 & $0.64(0.35,1.19)$ & $0.97(0.60,1.56)$ \\
\hline Quartile 3 & $1.33(0.77,2.30)$ & $0.94(0.59,1.50)$ \\
\hline Quartile 4 & $0.91(0.49,1.68)$ & $1.20(0.76,1.89)$ \\
\hline p-trend & 0.6041 & 0.4393 \\
\hline \multicolumn{3}{|l|}{ Serum sICAM-1 quartiles $\dagger(n=1045)$} \\
\hline Quartile 1 & 1 (referent category) & 1 (referent category) \\
\hline Quartile 2 & $1.15(0.65,2.04)$ & $0.96(0.61,1.50)$ \\
\hline Quartile 3 & $1.28(0.72,2.27)$ & $0.94(0.61,1.46)$ \\
\hline Quartile 4 & $1.26(0.69,2.30)$ & $0.90(0.56,1.45)$ \\
\hline p-trend & 0.4112 & 0.6593 \\
\hline
\end{tabular}

* sVCAM-1: soluable vascular cell adhesion molecule-1; sVCAM Quartiles: Quartile 1 (0-638.8 (ng/ml)), Quartile 2 (638.9-755.2 ng/ml), Quartile 3 (755.3-878.7), Quartile 4 ( > 878.7 ng/ml)

$\uparrow$ sICAM-1: soluable intercellular adhesión molecule-1; sICAM Quartiles: Quartile 1 (0-234.2 (ng/ml)), Quartile 2 (234.3-278.4 ng/ml), Quartile 3 (278.5-324.6), Quartile 4 (>342.6 ng/ml)

\$Adjusted for age (years), education categories (<high school, high school, $>$ high school), smoking (never, former, current), alcohol intake (never, former, current), body mass index $\left(\mathrm{kg} / \mathrm{m}^{2}\right)$, hypertension (absent, present) and serum cholesterol (\%) 
Table 4. 4. Association between increasing serum levels of sVCAM-1 and sICAM-1 and incident CKD stratified by hypertension status

\begin{tabular}{|c|c|c|}
\hline & $\begin{array}{c}\text { No Hypertension } \\
(\mathrm{n}=590)\end{array}$ & $\begin{array}{l}\text { Hypertension } \\
(\mathrm{n}=455)\end{array}$ \\
\hline Serum sVCAM-1 quartiles*(n=1045) & $\begin{array}{c}\text { Multivariable-adjusted } \\
\text { hazards ratio }(95 \% \text { confidence interval })\end{array}$ & $\begin{array}{c}\text { Multivariable-adjusted } \\
\text { hazards ratio }(95 \% \text { confidence interval }) \ddagger\end{array}$ \\
\hline Quartile 1 & 1 (referent category) & 1 (referent category) \\
\hline Quartile 2 & $0.73(0.41,1.30)$ & $0.92(0.52,1.62)$ \\
\hline Quartile 3 & $1.15(0.68,1.96)$ & $1.12(0.64,1.94)$ \\
\hline Quartile 4 & $1.10(0.62,1.95)$ & $1.83(0.69,2.04)$ \\
\hline $\mathrm{p}$-trend & 0.4589 & 0.4198 \\
\hline \multicolumn{3}{|l|}{ Serum sICAM-1 quartiles $\dagger(n=1045)$} \\
\hline Quartile 1 & 1 (referent category) & 1 (referent category) \\
\hline Quartile 2 & $1.39(0.81,2.39)$ & $0.79(0.46,1.36)$ \\
\hline Quartile 3 & $0.82(0.45,1.45)$ & $1.21(0.72,2.03)$ \\
\hline Quartile 4 & $1.29(0.72,2.32)$ & $0.85(0.48,1.51)$ \\
\hline p-trend & 0.7463 & 0.9415 \\
\hline
\end{tabular}

* sVCAM-1: soluable vascular cell adhesion molecule-1; sVCAM Quartiles: Quartile 1 (0-638.8 (ng/ml)), Quartile 2 (638.9-755.2 ng/ml), Quartile 3 (755.3-878.7), Quartile 4 ( > 878.7 ng/ml)

†sICAM-1: soluable intercellular adhesión molecule-1; sICAM Quartiles: Quartile 1 (0-234.2 (ng/ml)), Quartile $2(234.3-278.4 \mathrm{ng} / \mathrm{ml})$, Quartile 3 (278.5-324.6), Quartile 4 (>342.6 ng/ml)

\$Adjusted for age (years), education categories (<high school, high school, > high school), smoking (never, former, current), alcohol intake (never, former, current), body mass index $\left(\mathrm{kg} / \mathrm{m}^{2}\right)$, and serum cholesterol $(\%)$ 


\section{Chapter 5}

Manuscript 4: The Association of Markers of Endothelial Dysfunction and Cardiovascular Mortality 


\subsection{INTRODUCTION}

Cardiovascular disease is the leading cause of mortality in the United States. ${ }^{149}$ Endothelial dysfunction is a key pathophysiological process involved in the development of cardiovascular disease ${ }^{76}$ Soluble adhesion molecules, including serum levels of soluble vascular cell adhesion molecule-1 (sVCAM) and intercellular adhesion molecule -1 (sICAM) have been used as reliable markers of endothelial dysfunction in epidemiological studies. ${ }^{150}$ sVCAM-1 and sICAM-1 levels have shown to be associated with cardiovascular disease in the general population $^{114,151}$ and have also been shown to be associated with cardiovascular mortality in subjects undergoing hemodialysis ${ }^{91}$, and those with coronary heart disease ${ }^{92}$ and rheumatoid $\operatorname{arthritis}^{93}$. However, to our knowledge, no epidemiological study has examined the putative relationship between soluble adhesion molecules and cardiovascular mortality in the general population. Therefore, we examined serum levels of sVCAM-1 and sICAM-1 in relationship to cardiovascular mortality in a population-based sample of adults from Wisconsin. 


\subsection{METHODS}

\subsubsection{Population}

The current study is based on data from the Beaver Dam Eye study, a population-based cohort study in Wisconsin originally aimed at studying age-related eye diseases. The methods

used to identify and describe the Beaver Dam population have appeared in previous reports. ${ }^{152,153}$ In brief, a private census of the population of Beaver Dam, Wisconsin, was performed from September 1987 to May 1988 to identify all residents in the city or township of Beaver Dam who were 43-84 years of age. Of the 5,924 eligible individuals (98\% Caucasians), 4,926 (83.1\%) participated in the baseline examination between March 1, 1988 and September 14, 1990. Comparisons between participants and nonparticipants at the time of the baseline examination have appeared elsewhere ${ }^{153}$.

The baseline examination was followed by four follow-up examinations every 5 years. Written informed consent was obtained from each subject at each examination. The study was approved by the Human Subjects Committee of the University of Wisconsin School of Medicine and Public Health, Madison, WI. Serum levels of sVCAM-1 and sICAM -1 were measured on a random sample of the baseline cohort $(\mathrm{n}=1793)$ as part of an ancillary study examining risk factors for kidney disease.

For the longitudinal analyses with SVCAM-1 and sICAM-1 as the main exposure, out of 1793 individuals with available sVCAM-1 and sICAM-1 measurements, we excluded subjects with missing information on covariates included in the multivariable model $(n=38)$ and those who had a history of prevalent cardiovascular disease $(\mathrm{n}=247)$. This resulted in $\mathrm{n}=1508$ cardiovascular disease-free individuals with complete covariate information to form the at-risk study population. 


\subsubsection{Exposure ascertainment}

The baseline and follow-up examinations included measurement of weight, height, systolic and diastolic blood pressure by trained observers and administering a standardized questionnaire that collected information regarding participants' demographic characteristics, details regarding cigarette smoking, alcohol intake, medical histories and medications taken, including physician-diagnosed diabetes, hypertension or cardiovascular disease (CVD). Nonfasting blood specimens were obtained for measurement of plasma glucose, glycosylated hemoglobin, serum total cholesterol and high density lipoprotein (HDL) cholesterol.

Age was defined as the participants' age at the time of the baseline examination. Education was categorized as less than high school, high school, or beyond high school. Body mass index (BMI) was defined as participants' weight in kilograms divided by their height in meters squared. Hypertension was defined as a systolic blood pressure of $140 \mathrm{mmHg}$ or higher, and/or a diastolic blood pressure $90 \mathrm{mmHg}$ or higher, and/or the combination of self-reported hypertension diagnosis by a physician and use of antihypertensive medications. Diabetes was defined as being treated with insulin, oral hypoglycemic agents, or were newly classified as having diabetes based on the glycosylated hemoglobin value that was $\geq 6.5 \%$, consistent with recent American Diabetes Association guidelines ${ }^{104}$ Chronic kidney disease was defined as having chronic kidney disease were defined as having an estimated glomerular filtration rate (GFR) of $<60 \mathrm{ml} / \mathrm{min}$ per $\mathrm{m}^{2}$, estimated from serum creatinine employing the re-expressed Modification of Diet in Renal Diseases (MDRD) study equation. ${ }^{117}$

At cohort examinations, blood samples were collected in three 10-mL liquid EDTA blood tubes, placed on ice packs, stored in Styrofoam containers until they were centrifuged and aliquoted for storage in freezers at $-80^{\circ} \mathrm{C}$ until the time of laboratory analysis. Quality control 
samples were routinely frozen with study participant samples. Baseline frozen plasma samples were randomly ordered (to further reduce systematic bias and inter assay variation) and thawed and batch-analyzed for markers of endothelial function (sVCAM-1, sICAM-1).

sVCAM-1 was measured in plasma or serum using the quantitative sandwich enzyme technique of the enzyme-linked immunosorbent assay (ELISA) Parameter kit from R \& D Systems (Minneapolis, MN). The intensity of the color was measured on a SpectraMax spectrophotometer (Molecular Devices, Sunnyvale, California). The inter-assay CV range was $8.9-10.2 \%$

sICAM-1 (was measured in plasma using the quantitative sandwich enzyme technique of the enzyme-linked immunosorbent assay (ELISA) Parameter kit from R \& D Systems (Minneapolis, MN). The intensity of the color was measured on a SpectraMax spectrophotometer (Molecular Devices, Sunnyvale, California). The inter-assay CV range was $6.0-10.1 \%$.

\subsubsection{Outcome of Interest: Cardiovascular Mortality}

We ascertained mortality between the baseline examination and December 31, 2002. Mortality surveillance of the cohort consisted of reviews of daily newspaper obituaries, regular contact with study participants and their relatives, designated contact persons, or physicians. Deaths were confirmed with death certificate data from annual requests made to the Wisconsin Center for Health Statistics, Section of Vital Statistics. The names of persons who have moved out of Wisconsin and those who had been lost to follow-up or were suspected to have died were submitted for matching against Wisconsin death records and the National Death Index. For each match made, a copy of the death certificate was secured from the appropriate state. Only deaths 
that have been confirmed by death certificates were included in the definition of mortality. Death certificates were collected and coded by trained nosologists using the International Classification of Diseases, Ninth Revision (ICD-9). We have estimated that all the deaths in the cohort have been identified by our surveillance methods. Cardiovascular mortality was identified using ICD 9 codes 401-459, including death due to coronary heart disease and stroke as the underlying cause of death.

\subsubsection{Statistical methods}

We were interested in the association between serum levels of sVCAM-1 and SICAM-1 and cardiovascular mortality. We categorized sVCAM-1 and sICAM-1 into quartiles for the main analysis. We also analyzed these markers as continuous variables after logarithmic transformation due to their skewed distribution.

We used chi-square test and analysis of variance to compare the relationship of selected baseline characteristics to increasing categories of sVCAM-1. We used multivariable Cox proportional hazard regression models to determine the hazard ratio (HR) and 95 percent confidence interval (CI) of 15-year cardiovascular mortality, controlling simultaneously for potential confounders. We used two nested Cox proportional hazard regression models: an unadjusted model and a multivariable-adjusted model, adjusted for age (years), education categories (<high school, high school, > high school), smoking (never, former, current), alcohol

intake (never, former, current), body mass index $\left(\mathrm{kg} / \mathrm{m}^{2}\right)$, hypertension (absent, present), diabetes (absent, present), serum cholesterol (\%) and chronic kidney disease (yes, no). To examine the consistency of the association between SVCAM-1 and sICAM-1 levels and cardiovascular mortality, we performed subgroup analyses by gender (men, women), diabetes mellitus (absent, 
present), hypertension (absent, present) and chronic kidney disease (absent, present). Finally, we evaluated effect modification by including cross-product multiplicative interaction terms in regression models (alpha $=0.10$ for testing interaction). 


\subsection{RESULTS}

Among 1508 study participants included in the current analysis, there were 639 men and 869 women. Overall, 212 subjects (14\%) died of cardiovascular causes, including 96 men and 116 women.

Table 5.1 presents the characteristics of the study population by increasing serum sVCAM-1 levels at baseline. Those who had higher serum sVCAM-1 levels were more likely to be older, educated below high school, never smokers, never drinkers, have lower cholesterol levels and diastolic blood pressures.

Table 5.2 presents the association between increasing categories of serum sVCAM-1, sICAM-1 and cardiovascular mortality. The multivariable model for sVCAM-1 showed a positive association between increasing serum sVCAM-1 levels and cardiovascular mortality, although the model evaluating linear trend in this association was just shy of statistical significance ( $\mathrm{p}$-trend=0.0904). The multivariable model for sICAM-1 showed a positive association between increasing serum sICAM-1 levels and cardiovascular mortality and the model evaluating linear trend in this association was statistically significant ( $\mathrm{p}$-trend $=0.0097$ ).

Table 5.3 presents the associations between increasing categories of serum SVCAM-1, sICAM-1 and cardiovascular mortality, stratified by gender, diabetes, hypertension, chronic kidney disease and BMI status. The multivariable model for sVCAM-1 showed a stronger association with cardiovascular mortality in women, those with diabetes, hypertension, chronic kidney disease and those with normal BMI. The multivariable model for sICAM-1 showed a stronger association with cardiovascular mortality in women, in those without diabetes and hypertension and in those with chronic kidney disease and normal BMI. Formal statistical tests for interaction were not significant (all p-interactions $>0.10$ ). 
Supplementary analyses that additionally adjusted for high sensitivity c-reactive in the multivariable model as a marker of inflammation did not alter the magnitude of results. Compared to the lowest quartiles of sVCAM-1 (referent) and sICAM-1 (referent), the multivariable odds ratio (95\% confidence interval) of cardiovascular mortality for sVCAM-1 quartiles $1-4$ respectively were $1.34(0.80,2.24), 1.26(0.77,2.08)$, and $1.53(0.94,2.51))$ and for sICAM-1 quartiles 1-4 respectively were $1.39(0.89,2.17), 1.42(0.92,2.20)$ and $1.73(1.13,2.76)$. 


\subsection{DISUCSSION}

In a population-based sample of adults from Wisconsin, increasing serum levels of sVCAM-1 and sICAM-1 were found to be positively associated with cardiovascular mortality. These associations were independent of age, gender smoking, alcohol intake, education, hypertension, diabetes, chronic kidney disease, body mass index and total cholesterol. In subgroup analyses, the association between sVCAM-1 and sICAM-1 levels and cardiovascular mortality appeared to be relatively consistently present among those with and without diabetes, hypertension, chronic kidney disease and obesity.

Serum levels of sICAM-1 and sVCAM-1 have been used in epidemiological research before as reliable markers of endothelial dysfunction. ${ }^{150}$ Previous studies have reported that markers of endothelial dysfunction are associated with increased risk cardiovascular disease $^{76,114,151}$ and cardiovascular mortality in ill subpopulations. ${ }^{91-93}$ However, since these endothelial dysfunction markers are also known to be elevated secondary to these chronic diseases (or as a result of treatments for these diseases, such as hemodialysis for kidney disease), results from such studies of patient cohorts may not reflect disease processes occurring in the general population. In the current study, we examined, to our knowledge for the first time, the association between increasing levels of markers of endothelial dysfunction in serum and their relationship to cardiovascular mortality in a general population sample. Subsequently, we found that markers of endothelial dysfunction are positively related to cardiovascular mortality. This finding is consistent with a previous report of an overall association of markers of endothelial dysfunction and incident cardiovascular disease in the Atherosclerosis Risk in Communities Study. ${ }^{114}$ 
Because there are several cardiovascular risk factors that are associated with an increase in endothelial dysfunction, including gender ${ }^{154,155}$, diabetes, ${ }^{156,157}$ hypertension, ${ }^{157,158}$ chronic kidney disease $\mathrm{e}^{159,160}$ and obesity ${ }^{161,162}$, we adjusted and stratified by these risk factors to test the independent association between these markers of endothelial dysfunction and cardiovascular mortality. We found that the association between endothelial dysfunction and cardiovascular mortality was attenuated, but still present, even after multivariable adjustment for confounders. Also, there was no statistically significant difference in the association of markers of endothelial dysfunction to cardiovascular mortality in subgroup analyses by gender, diabetes, hypertension, chronic kidney disease and BMI.

The intact, healthy endothelium secretes various cardio-protective substances including nitric oxide, which diffuses to surrounding tissues and cells, relaxing smooth-muscle cells and preventing platelet adhesion and aggregation, preventing expression of adhesion molecules, leukocyte adhesion and migration into the arterial wall and arterial smooth muscle cell proliferation ${ }^{77}$. Conversely, early stages of endothelial dysfunction is characterized by increased formation of reactive oxygen species and increased expression of adhesion molecules such as sVCAM-1 and sICAM-1, which can react with NO forming peroxynitrate ${ }^{78}$, reducing NO bioavailability, which over the long run may be involved in increased risk of cardiovascular disease $^{79}$. Results from our study, which show a positive association between increased circulating levels of endothelial adhesion molecules and cardiovascular mortality in a general population sample, are therefore consistent with these proposed biological mechanisms and indirectly suggest that there may be a role for treating endothelial dysfunction to prevent cardiovascular mortality. 
Strengths of this study include its population-based sample, high participation rate, use of standardized protocols for exposure and outcome measurement, complete mortality surveillance and the availability of specific markers of endothelial dysfunction, including SVCAM-1 and sICAM-1 levels. Also, misclassification of cardiovascular mortality may have biased our results. Study limitation include selective survival, high variability and the generalizability of our study to other populations as this sample is a $99 \%$ white and is greater than or equal to 43 years of age.

In conclusion, in a population-based sample of Wisconsin adults, we found that increasing serum levels of sVCAM-1 and sICAM-1 levels were positively associated with cardiovascular mortality, independent of age smoking status, alcohol intake, education, hypertension, diabetes, chronic kidney disease, BMI, and total cholesterol levels. 


\subsection{TABLES}

Table 5.1. Baseline characteristics by vascular cell adhesion molecule 1 (sVCAM -1) quartiles

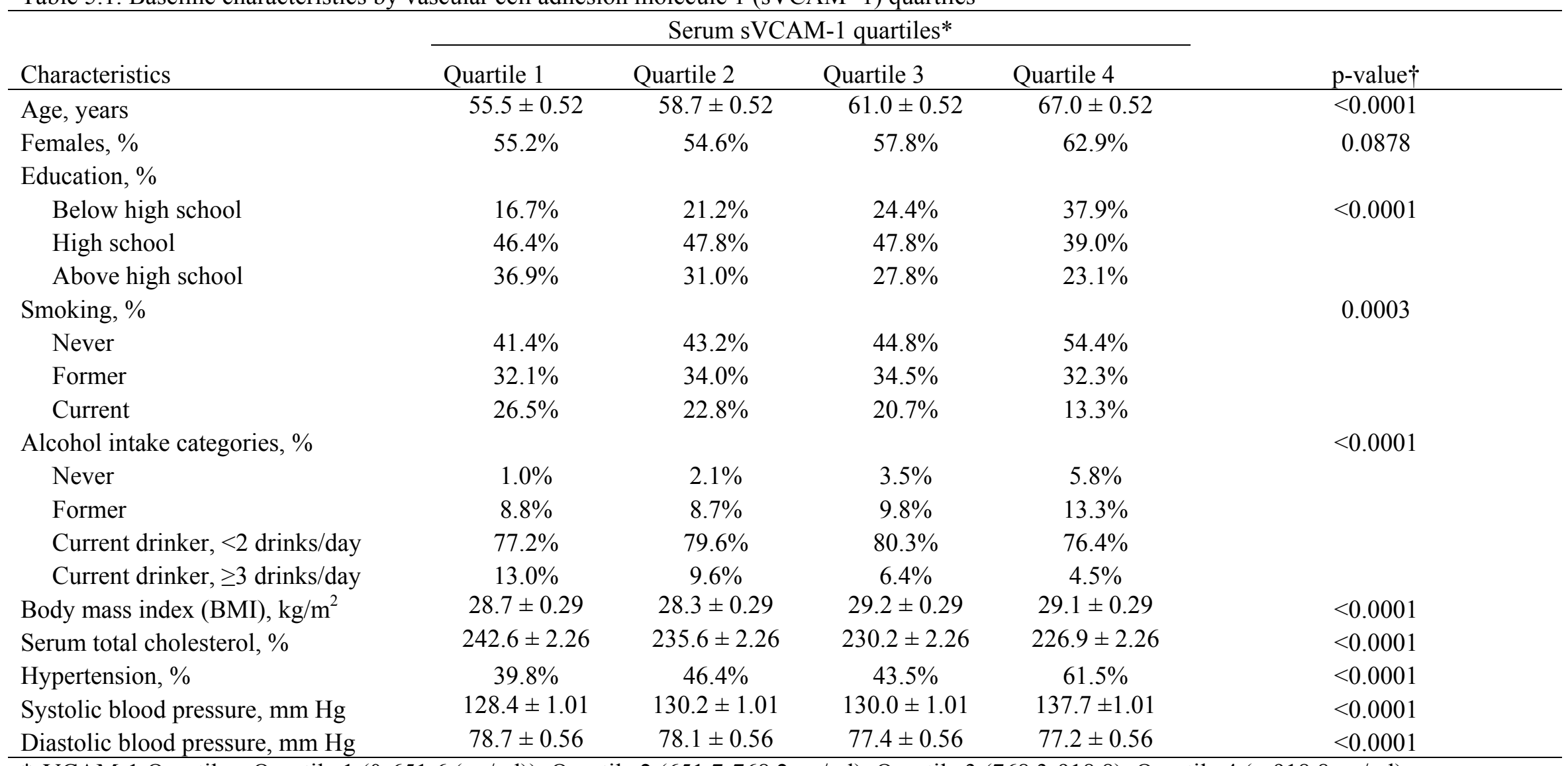

*sVCAM-1 Quartiles: Quartile 1 (0-651.6 (ng/ml)), Quartile 2 (651.7-768.2 ng/ml), Quartile 3 (768.3-918.8), Quartile 4 ( $>918.8 \mathrm{ng} / \mathrm{ml})$

$\dagger p$-value estimated by analysis of variance or chi-square test as appropriate 
Table 5.2. Association between increasing serum levels of sVCAM-1 and sICAM-1 and cardiovascular mortality

\begin{tabular}{lcccc}
\hline $\begin{array}{l}\text { Serum sVCAM-1 quartiles* } \\
(\mathrm{n}=1508)\end{array}$ & No. at risk & $\begin{array}{c}\text { Mortality } \\
\text { cases } \\
(\mathrm{n}=212)\end{array}$ & $\begin{array}{c}\text { Unadjusted hazards ratio }(95 \% \\
\text { confidence interval) }\end{array}$ & $\begin{array}{c}\text { Multivariable-adjusted } \\
\text { hazards ratio (95\% confidence interval) } \ddagger\end{array}$ \\
\hline Quartile 1 & 377 & 24 & 1 (referent category) & 1 (referent category) \\
Quartile 2 & 377 & 39 & $1.68(1.01,2.79)$ & $1.31(0.78,2.19)$ \\
Quartile 3 & 377 & 54 & $2.40(1.48,3.88)$ & $1.22(0.74,2.01)$ \\
Quartile 4 & 377 & 95 & $4.90(3.07,7.52)$ & $1.56(0.95,2.54)$ \\
p-trend & & & $<0.0001$ & 0.0904
\end{tabular}

Serum sICAM-1 quartiles $\dagger$

$(\mathrm{n}=1504)$

Quartile 1

$377 \quad 31$

1 (referent category)

1 (referent category)

Quartile 2

378

$1.69(1.09,2.63)$

$1.45(0.93,2.26)$

Quartile 3

372

$2.01(1.31,3.10)$

$1.47(0.95,2.27)$

Quartile 4

377

59

$2.47(1.62,3.76)$

$1.79(1.17,2.76)$

p-trend

$<0.0001$

0.0097

*sVCAM-1 Quartiles: Quartile 1 (0-651.6 (ng/ml)), Quartile 2 (651.7-768.2 ng/ml), Quartile 3 (768.3-918.8), Quartile 4 ( $>918.8 \mathrm{ng} / \mathrm{ml})$ $\uparrow$ sICAM-1 Quartiles: Quartile 1 (0-237.2 (ng/ml)), Quartile 2 (237.3-281.2 ng/ml), Quartile 3 (281.3-330.8), Quartile 4 ( > 330.8 ng/ml) ¥Adjusted for age (years), education categories (<high school, high school, $>$ high school), smoking (never, former, current), alcohol intake (never, former, current), body mass index ( $\left.\mathrm{kg} / \mathrm{m}^{2}\right)$, hypertension (absent, present), diabetes (absent, present), serum cholesterol (\%), chronic kidney disease (yes, no) 
Table 5.3. Association by sICAM-1 and sVCAM-1 and cardiovascular mortality in selected subgroups*

\section{sVCAM-1}

The multivariable-adjusted hazards ratio $\dagger(95 \%$

Sample confidence interval) for one standards deviation

size increaset.

\section{sICAM-1}

The multivariable-adjusted hazards ratio $\dagger(95 \%$ confidence interval) for one standards deviation increase

Categories

Female

869

\section{Men}

$.51(1.18,1.95)$

$1.25(1.00,1.56)$

$1.54(1.18,2.01)$

Body Mass Index

Normal BMI

1144

Overweight/Obese

Diabetes mellitus

$\begin{array}{ll}\text { Absent } & 1020 \\ \text { Present } & 488\end{array}$

$$
1.59(1.11,2.27)
$$$$
1.34(1.08,1.67)
$$

$1.68(1.09,2.60)$

$1.13(0.92,1.35)$

$1.60(1.09,2.32)$

$1.14(0.96,1.36)$

$1.36(1.13,1.64)$

$1.21(0.93,1.58)$

Hypertension

$\begin{array}{lll}\text { Absent } & 560 & 1.28(0.95,1.73) \\ \text { Present } & 948 & 1.39(1.11,1.73)\end{array}$

Chronic kidney disease

$$
\text { Absent }
$$

268
$1.35(1.06,1.70)$

$1.41(1.12,1.96)$
$1.88(1.25,2.83)$

$1.08(0.90,1.30)$

$1.18(0.98,1.43)$

$1.37(1.08,1.75)$

*All p-interactions $>0.10$

$\uparrow$ Adjusted for age (years), gender (male, female), education categories (<high school, high school, >high school), smoking (never, former, current), alcohol intake (never, former, current), body mass index $\left(\mathrm{kg} / \mathrm{m}^{2}\right)$, hypertension (absent, present), diabetes (absent, present), serum cholesterol (\%), chronic kidney disease (yes, no)

$\$$ one standard deviation sVCAM-1 = 288.4 units; one standard deviation of sICAM-1 = 98.5 units 
Chapter 6

Discussion 


\subsection{SUMMARY OF KEY FINDINGS}

In an attempt to resolve the current gaps in literature (as mentioned in the Introduction section), we conducted four related studies in a large population-based cohort study in Beaver Dam, Wisconsin, to examine the role of serum markers of endothelial dysfunction on various common metabolic and cardiovascular outcomes in humans, including diabetes mellitus, hypertension, $\mathrm{CKD}$, and cardiovascular mortality. We found that markers of endothelial dysfunction, including serum sVCAM-1 and sICAM-1, were positively associated with the risk of developing diabetes, hypertension, and cardiovascular mortality, but not with CKD (see a summary of these findings in Table 6.1.). Further, in subgroup analyses, we found that the association between these endothelial dysfunction markers and diabetes mellitus was present mainly in men, but not women. All other associations were present similarly in men and women. Based on these findings, we propose a conceptual model that markers of endothelial dysfunction are related to cardiovascular mortality probably through its relation to diabetes mellitus and hypertension, but not to CKD (see Figure 6.1.).

\subsection{ENDOTHELIAL DYSFUNCTION AND DIABETES MELLITUS (MANUSCRIPT 1/CHAPTER 2)}

The findings from our first manuscript of an association between sVCAM-1 and sICAM1 and diabetes mellitus in men are consistent with a previous study by Thorand et al. showing serum endothelial dysfunction marker levels to be associated with increased risk of type 2 diabetes in men and not in women. ${ }^{84}$ Also, another study showed that when measuring endothelial function as reactive hyperemia by peripheral arterial tonometry, endothelial dysfunction was associated with diabetes and hyperglycemia in adolescent males but not 
adolescent females. ${ }^{83}$ It is biologically plausible that endothelial dysfunction is related to incident diabetes mellitus through its relation to mechanisms such as hyperglycemia, insulin resistance, excess free fatty acids release and compensatory hyperinsulinemia. ${ }^{105}$ Similarly, several plausible mechanisms can be postulated to explain the lack of association between endothelial dysfunction and diabetes mellitus in women in the current study, including that female hormones are cardioprotective and therefore to some extent may mitigate any adverse effect of endothelial dysfunction. ${ }^{106}$ However, there is a need for more studies in this topic to clarify the modifying effect of female gender in the association between endothelial dysfunction and diabetes.

\subsection{ENDOTHELIAL DYSFUNCTION AND HYPERTENSION (MANUSCRIPT}

\section{2/CHAPTER 3)}

The findings from our second manuscript of an association between sVCAM-1 and hypertension is consistent with one previous prospective epidemiological study examining the relationship between biomarkers of endothelial dysfunction and hypertension in those with type 1 diabetes ${ }^{86}$ and another cross-sectional study in the general population conducted in China. ${ }^{88}$ The observed association between endothelial dysfunction and hypertension may be due to endothelial damage and related dysfunction impairing the vasodilator mechanism of endothelium-derived relaxing factor (nitric oxide), subsequently causing elevated blood pressure $^{34}$. Endothelial dysfunction may also be related to other mechanisms involved in the development of hypertension, including insulin resistance, ${ }^{64}$ systemic inflammation ${ }^{65}$ and hyperuricemia. ${ }^{66}$ However, in our study, we also found that even though sVCAM-1 was positively related to incident hypertension, sICAM-1 was not. While the reasons for these differing associations between SVCAM-1 and sICAM-1 are not entirely clear, there are several 
hypotheses that can be postulated to explain this finding. It is possible that even though these adhesion molecules are similar in structure they have different functions. For example, recent animal studies have shown that VCAM-1, and not ICAM-1, plays a major role in the initiation of atherosclerosis $^{125}$, and others that have reported differential expression patterns for these adhesion molecules in the vascular tree with VCAM-1 being expressed more in atherosclerotic lesions whereas ICAM-1 expression extending into uninvolved aorta and lesion protected regions. ${ }^{126}$ However, there is a need for more human studies to validate or refute our findings.

\subsection{ENDOTHELIAL DYSFUNCTION AND CKD (MANUSCRIPT 3/CHAPTER 4)}

The findings from our third manuscript showed that sVCAM-1 and sICAM-1 are not independently related to incident CKD. We originally initiated this study because there was strong biologically plausible data from animal studies to suggest that endothelial dysfunction may have a role in the development of kidney disease. Janssen et al. ${ }^{145}$ reported that ICAM-1 knockout mice developed significantly less severe experimentally induced nephrotoxic nephritis and glomerular crescents compared to control mice. Similarly, Kawasaki et al. showed that injection of antibodies against ICAM-1 prevented glomerular injury in experimental crescentic glomerular nephritis ${ }^{146}$. The importance of our study is that to our knowledge, this is the first prospective study in humans to examine the association between endothelial dysfunction and the development of renal disease using circulating levels of cellular adhesion molecules. Since markers of endothelial dysfunction are known to be elevated secondarily in kidney disease ${ }^{89,90}$ and also as a result of hemodialysis ${ }^{163}$, the first line of treatment for late stages of kidney disease, it is critically important to conduct a prospective study to investigate this association so as to clarify the temporal nature of any associations detected. In our study, we found that even though 
there was a moderate positive association between SVCAM-1 and sICAM-1 and incident CKD in the unadjusted model, the association was no longer present after adjusting for lifestyle and medical risk factors. Therefore, our findings indirectly suggest that treatment/control of lifestyle and medical risk factors for CKD such as higher BMI, diabetes, and hypertension should continue to be the mainstay of treatment for CKD.

\subsection{ENDOTHELIAL DYSFUNCTION AND CARDIOVASCULAR MORTALITY (MANUSCRIPT 4/CHAPTER 5)}

The findings from our fourth manuscript showed that sVCAM-1 and sICAM-1 levels are positively associated with cardiovascular mortality. Previous studies have reported that markers of endothelial dysfunction are associated with increased risk of developing cardiovascular disease $^{76,114,151}$ and cardiovascular mortality in ill subpopulations. ${ }^{91-93}$ However, since these endothelial dysfunction markers are also known to be elevated secondary to these chronic diseases $^{89,90,163}$ (or as a result of treatments for these diseases), results from such studies of patient cohorts may not reflect disease processes occurring in the general population. In the current study, we found that markers of endothelial dysfunction are positively related to cardiovascular mortality. Our finding is consistent with a previous report of an overall association of markers of endothelial dysfunction and incident cardiovascular disease in the Atherosclerosis Risk in Communities Study. ${ }^{114}$

\subsection{SIGNIFICANCE OF THE STUDY}

After exploring markers of endothelial dysfunction in relation to diabetes, hypertension, CKD and cardiovascular mortality, endothelial dysfunction was shown to play a major role in all 
chronic disease outcomes under study except CKD. Clinically, these results suggest that treating endothelial dysfunction may prevent diabetes, hypertension and cardiovascular mortality in patients and in populations.

\subsection{STRENGTHS AND LIMITATIONS}

Since from an epidemiological pint of view, temporality is a major tenant of a truly causal association, one important advantage of our study is the longitudinal nature of the Beaver Dam Eye Study cohort dataset. The availability of long-standing prospective data on a representative general population sample allowed us to elucidate the temporal nature of the association between markers of endothelial dysfunction and the various cardiometabolic outcomes we studied. This is especially important in the case of CKD where markers of

endothelial dysfunction are known to be elevated secondarily in later stages of kidney disease ${ }^{89,90}$ and also as a result of hemodialysis, a main stay of advanced CKD and end-stage renal disease treatment. ${ }^{163}$ Another advantage of our study is the availability of a truly population-based sample (as opposed to clinical patient populations). This is especially important in our study on cardiovascular morality as markers of endothelial dysfunction are known to be elevated secondarily in various disease states, such as those with rheumatoid arthritis and CKD. ${ }^{163}$ Other strengths of the Beaver Dam Eye Study cohort include its high participation rate and use of standardized protocols for exposure and outcome measurement and the availability of specific serum markers of endothelial dysfunction, including sVCAM-1 and sICAM-1 levels. Our results of positive and null associations between markers of endothelial dysfunction and incident chronic disease outcomes and cardiovascular mortality possess strong internal validity as we adjusted for and stratified by known confounders. 
A main study limitation is the limited generalizability of our study to other populations as our study sample is a $99 \%$ white sample. Also since we used serum markers of endothelial dysfunction, there is some potential for exposure misclassification which could under or overestimate our results. Finally, as in any longitudinal study, it is possible that there are biases related to selective survival (e.g. sick people dying more than healthy individuals), time-varying confounding (e.g. a smoker at baseline may quit smoking, whereas a nonsmoker may start smoking at a later date — which may affect their endothelial dysfunction marker level as well as risk of diseases). Assuming that these changes are randomly distributed across levels of baseline endothelial dysfunction marker, we believe that any misclassification is likely to be nondifferential and likely to underestimate the true association.

\subsection{FUTURE RESEARCH}

To build upon our novel findings, we suggest that future investigations should include assessing these relationships in ethnic diverse cohorts to assess whether or not the relationships differ by ethnicity. For example, it is possible that some of the observed associationsespecially the association between endothelial dysfunction and hypertension—may vary by race/ethnicity and that the difference in our findings to that in the literature may be explained by such underlying racial/ethnic differences. Thus there is a need for future studies with adequate sample sizes of various race/ethnicities to enable valid cross-ethnicity comparisons. Second, our

study measured these novel markers of endothelial dysfunction only in the baseline Beaver Dam Eye Study cohort study samples. Since it is well known that levels of these markers may change over time due to changing lifestyle, environmental, and biological factors, as a future research topic, it is important to study the time varying effect of these markers of endothelial dysfunction 
by measuring them at multiple follow-up occasions in the same cohort. Lastly, it is important to study the genetic factors that may predispose individuals to increased endothelial dysfunction in response to lifestyle and environmental risk factors.

\subsection{CONCLUSION}

In summary, markers of endothelial dysfunction, including serum levels of sVCAM-1 and sICAM-1 were found to be associated with increased risk of developing diabetes mellitus in men, and with hypertension and cardiovascular mortality in both men and women. However, these markers of endothelial dysfunction were not found to be associated with the risk of developing CKD, independent of known lifestyle and medical risk factors. Consequently, the significance of our findings is that we are able to clarify the relationships between endothelial dysfunction and various risk factors/mediators of cardiovascular mortality by examining them in a single, large, population-based dataset. Our results point to the conclusion that endothelial dysfunction may be related to cardiovascular mortality through its relation to diabetes mellitus and hypertension but not to CKD. 


\subsection{TABLES AND FIGURES}

\begin{tabular}{|l|c|c|c|c|c|c|c|}
\hline \multicolumn{1}{|c|}{ Table 6.1. Summary of Associations between Markers of Endothelial Dysfunction and Outcomes of Interest } \\
\hline & \multicolumn{3}{|c|}{ sVCAM-1 } & \multicolumn{3}{c|}{ sICAM-1 } \\
\hline & Overall & Men & Women & Overall & Men & Women \\
\hline Diabetes Mellitus & + & + & - & & + & + & - \\
\hline Hypertension & + & + & + & & - & - & - \\
\hline Chronic Kidney Disease & - & - & - & & - & - & - \\
\hline Cardiovascular Mortality & + & - & + & & + & - & - \\
\hline
\end{tabular}




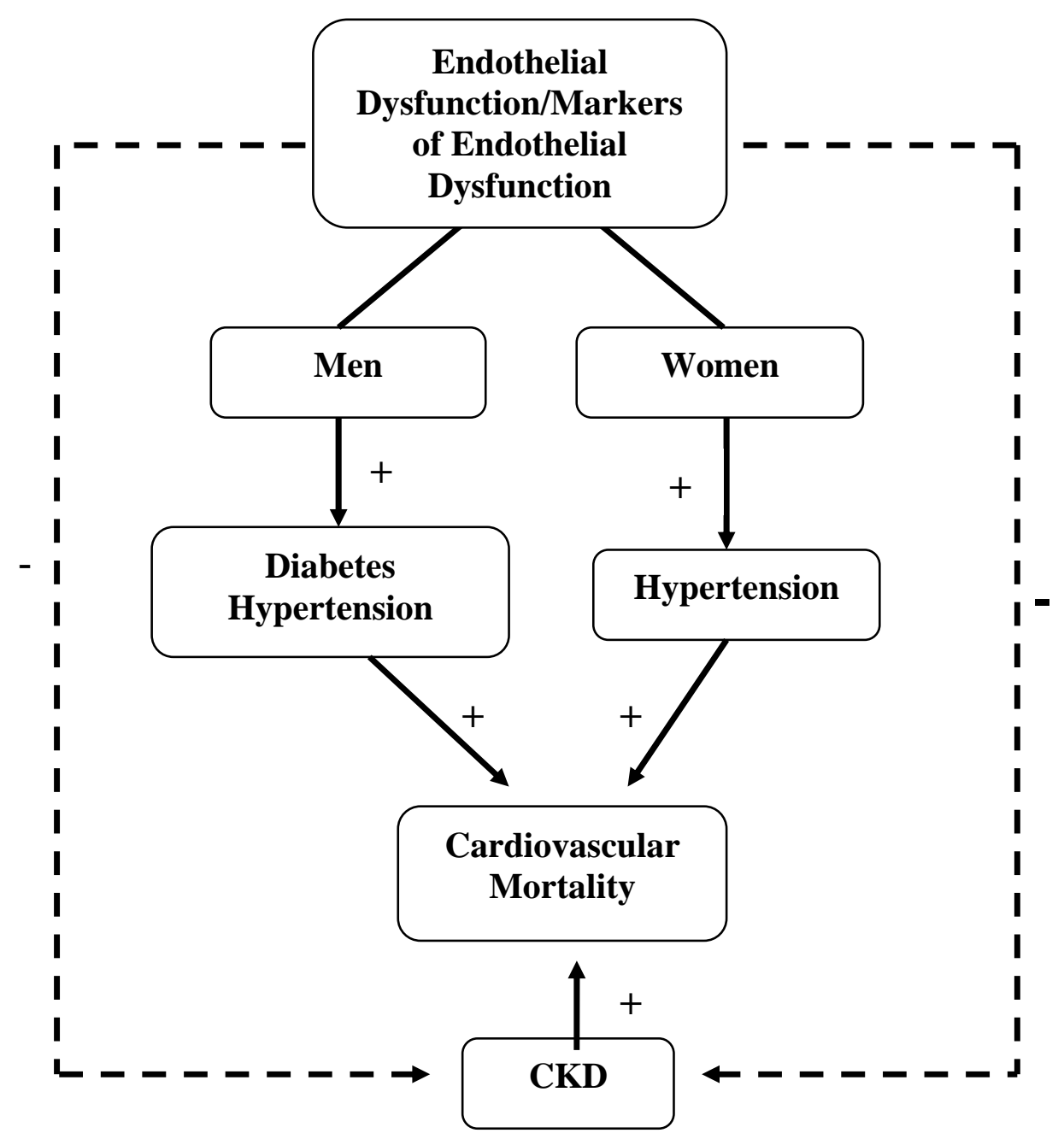

Figure 6. 1. The Mediation of Endothelial Dysfunction and Cardiovascular Mortality by Gender Based on results from the four studies, we propose that endothelial dysfunction is related to cardiovascular mortality through its relation to diabetes and hypertension in men, and through it relation to hypertension in women, since endothelial dysfunction was not found to be related to diabetes in them. Even though it is known that CKD is a risk factor for cardiovascular mortality, we propose that CKD is not involved in the causal association between endothelial dysfunction and cardiovascular mortality as we did not find an association between endothelial dysfunction and CKD. 


\section{References}

1. Centers for Disease Control and Prevention Deaths and Mortality: general information and national estimates on deaths and mortality in the United States, 2007. U S Department of Health and Human Services, Centers for Disease Control and Prevention.National Center for Health Statistics . 2008.

Ref Type: Journal (Full)

2. Wald N, Simmonds M, Morris J. Screening for future cardiovascular disease using age alone compared with multiple risk factors and age. PloS One. 2011;6:e18742.

3. Archer E, Blair S. Physical activity and the prevention of cardiovascular disease: from evolution to epidemiology. Prog Cardiovasc Dis. 2011;53:387-96.

4. Carnethon, MR, Biggs, ML, Barzilay, J, Kuller, LH, Mozaffarian, D, Mukamal, K, Smith, NL, and Siscovick, D. Diabetes and coronary heart disease as risk factors for mortality in older adults. Am J Med 123(6), 556.e1-559. 2010.

Ref Type: Journal (Full)

5. Kraja A, Hunt S, Rao D, Dávila-Román V, Arnett D, Province M. Genetics of hypertension and cardiovascular disease and their interconnected pathways: lessons from large studies. Curr Hypertens Rep. 2011;13:46-54.

6. Suárez García, FM, Pérez Martín, A, Peiró Moreno, S, and García García, FJ. Risk factors for 4-year mortality in older adults. Toledo Study. Rev Esp Geriatr Gerontol 43(2), 76-84. 2008.

Ref Type: Journal (Full) 
7. Caballero AE. Endothelial dysfunction in obesity and insulin resistance: a road to diabetes and heart disease. Obes Res. 2003;11:1278-89.

8. Nathan D, Cleary P, Backlund J, Genuth S, Lachin J, Orchard T et al. Intensive diabetes treatment and cardiovascular disease in patients with type 1 diabetes. N Engl J Med. 2005;353:2643-53.

9. Centers for Disease Control and Prevention. National diabetes fact sheet: general information and national estimates on diabetes in the United States, 2007. U S Department of Health and Human Services, Centers for Disease Control and Prevention Atlanta, GA 2008. 2008.

10. Creatore M, Moineddin R, Booth G, Manuel D, DesMeules M, McDermott S et al. Age- and sex-related prevalence of diabetes mellitus among immigrants to Ontario, Canada. CMAJ. 2011;182:781-89.

11. Knerr I, Wolf J, Reinehr T, Stachow R, Grabert M, Schober E et al. DPV Scientific Initiative of Germany and Austria. The 'accelerator hypothesis': relationship between weight, height, body mass index and age at diagnosis in a large cohort of 9,248 German and Austrian children with type 1 diabetes mellitus. Diabetologia. 2005;48:2501-4.

12. Everett C, Frithsen I. Evidence that prehypertension is a risk factor for Type 2 diabetes. Expert Rev Cardiovasc Ther. 2010;8:335-37. 
13. Bouguerra R, Alberti H, Smida H, Salem L, Rayana C, El Atti J et al. Waist circumference cut-off points for identification of abdominal obesity among the tunisian adult population. Diabetes Obes Metab. 2007;9:859-68.

14. Elhayany A, Lustman A, Abel R, Attal-Singer J, Vinker S. A low carbohydrate Mediterranean diet improves cardiovascular risk factors and diabetes control among overweight patients with type 2 diabetes mellitus: a 1-year prospective randomized intervention study. Diabetes Obes Metab. 2010;12:204-9.

15. Chobanian A, Bakris G, Black H, Cushman W, Green L, Izzo J et al. The Seventh Report of the Joint National Committee on Prevention, Detection, Evaluation, and Treatment of High Blood Pressure. Hypertension. 2003;42:1206-52.

16. Egan B, Zhao Y, Axon N. US Trends in Prevalence, Awareness, Treatment, and Control of Hypertension, 1988-2008. JAMA. 2010;303:2043-50.

17. Bezerra P, Leão M, Queiroz J, Melo E, Pereira F, Nóbrega M et al. Family history of hypertension as an important risk factor for the development of severe preeclampsia. Acta Obstet Gynecol Scand. 2010;89:612-17.

18. Diez Roux A, Chambless L, Merkin S, Arnett D, Eigenbrodt M, Nieto FJ et al. Socioeconomic disadvantage and change in blood pressure associated with aging. Circulation. 2002;106:703-10.

19. Chou P, Chen C, Chen H, Chang M. Epidemiology of isolated systolic hypertension in Pu-Li, Taiwan. Int J Cardiol. 1992;35:219-26. 
20. Arici M, Turgan C, Altun B, Sindel S, Erbay B, Derici U et al. Hypertension incidence in Turkey (HinT): a population-based study. J Hypertension. 2010;28:240-244.

21. Ji H, Zheng W, Wu X, Liu J, Ecelbarger C, Watkins R et al. Sex chromosome effects unmasked in angiotensin II-induced hypertension. Hypertension. 2010;55:1275-82.

22. Juhász M, Katona E, Settakis G, Paragh G, Molnár C, Fülesdi B et al. Gender-related differences in adolescent hypertension and in target organ effects. J Womens Health (Larchmt). 2010;19:759-65.

23. Arakawa K. Hypertension and Exercise. Clin Exp Hypertens. 1993;15:1171-79.

24. Ibhazehiebo K, Dimkpa U, Iyawe VI. Hypertension, and blood pressure response to graded exercise in young obese and non-athletic Nigerian university students. Niger J Physiol Sci. 2007;22:37-42.

25. Staffileno B, Minnick A, Coke L, Hollenberg S. Blood pressure responses to lifestyle physical activity among young, hypertension-prone African-American women. J Cardiovasc Nurs. 2007;22:107-17.

26. Miura K, Okuda N, Turin T, Takashima N, Nakagawa H, Nakamura K et al. Dietary salt intake and blood pressure in a representative Japanese population: baseline analyses of NIPPON DATA80. J Epidemiology. 2010;20:S524-S530.

27. Dahl L, Love R. Evidence for relationship between sodium (chloride) intake and human essential hypertension. AMA Arch Intern Med. 1954;94:525-31. 
28. Dahl L, Love R. Etiological role of sodium chloride intake in essential hypertension in humans. J Am Med Assoc. 1957;164:397-400.

29. Leenen F, McInnia N, Fodor G. Obesity and the Prevalence and Management of Hypertension in Ontario, Canada. Am J Hypertension. 2010.

30. Grebla R, Rodriguez C, Borrell L, Pickering T. Prevalence and determinants of isolated systolic hypertension among young adults: the 1999-2004 US National Health And Nutrition Examination Survey. J Hypertension. 2010;28:15-23.

31. Hering D, Kucharska W, Kara T, Somers V, Narkiewicz K. Smoking is associated with chronic sympathetic activation in hypertension. Blood Pressure. 2010;19:152-55.

32. De Marco M, de Simone G, Roman M, Chinali M, Lee E, Russell M et al. Cardiovascular and metabolic predictors of progression of prehypertension into hypertension: the Strong Heart Study. Hypertension. 2009;54:974-80.

33. Wright J, Stevens J, Poole C, Flegal K, Suchindran C. The impact of differences in methodology and population characteristics on the prevalence of hypertension in US adults in 1976-1980 and 1999-2002. Am J Hypertension. 2010;23:620-626.

34. Van Zwieten P. Endothelial dysfunction in hypertension. A critical evaluation. Blood Press Suppl. 1997;2:67-70.

35. Sarnak M, Levey A, Schoolwerth A, Coresh J, Culleton B, Hamm L et al. Kidney disease as a risk factor for development of cardiovascular disease: a statement from the American Heart Association Councils on Kidney in Cardiovascular Disease, High 
Blood Pressure Research, Clinical Cardiology, and Epidemiology and Prevention. Circulation. 2003;108:2154-69.

36. Go A, Chertow G, Fan D, McCulloch E, su C. Chronic kidney disease and the risks of death, cardiovascular events, and hospitalization. N Engl J Med. 2004;351:1296-305.

37. Coresh J, Selvin E, Stevens L, Manzi J, Kusek J, Eggers P et al. Prevalence of chronic kidney disease in the United States. JAMA. 2007;298:2038-47.

38. Brancati FL, Whelton PK, Randall BL, Neaton JD, Stamler J, Klag MJ. Risk of endstage renal disease in diabetes mellitus: a prospective cohort study of men screened for MRFIT. Multiple Risk Factor Intervention Trial. JAMA. 1997;278:2069-74.

39. Fox CS, Larson MG, Leip EP, Culleton B, Wilson PW, Levy D. Predictors of newonset kidney disease in a community-based population. JAMA. 2004;291:844-50.

40. Klag MJ, Whelton PK, Randall BL, Neaton JD, Brancati FL, Ford CE et al. Blood pressure and end-stage renal disease in men. N Engl J Med. 1996;334:13-18.

41. Perry Jr H, Miller J, Fornoff J, Baty J, Sambhi M, Rutan G et al. Early predictors of 15year end-stage renal disease in hypertensive patients. Hypertension. 1995;25:587-94.

42. Perneger TV, Nieto FJ, Whelton PK, Klag MJ, Comstock GW, Szklo M. A prospective study of blood pressure and serum creatinine. Results from the 'Clue' Study and the ARIC Study. JAMA. 1993;269:488-93.

43. Coresh J, Wei GL, McQuillan G, Brancati FL, Levey AS, Jones C et al. Prevalence of high blood pressure and elevated serum creatinine level in the United States: findings 
from the third National Health and Nutrition Examination Survey (1988-1994). Arch Intern Med. 2001;161:1207-16.

44. Haroun MK, Jaar BG, Hoffman SC, Comstock GW, Klag MJ, Coresh J. Risk factors for chronic kidney disease: a prospective study of 23,534 men and women in Washington County, Maryland. J Am Soc Nephrol. 2003;14:2934-41.

45. Coresh J, Astor B, Greene T, Eknoyan G, Levey A. Prevalence of chronic kidney disease and decreased kidney function in the adult US population: Third National Health and Nutrition Examination Survey. Am J Kidney Dis. 2003;41:1-12.

46. Iseki K, Ikemiya Y, Fukiyama K. Risk factors of end-stage renal disease and serum creatinine in a community-based mass screening. Kidney Int. 1997;51:850-854.

47. Vupputuri S, Sandler D. Lifestyle risk factors and chronic kidney disease. Annals of Epidemiology. 2003;13:712-20.

48. Blever A, Shemanski L, Burke G, Hansen J, Appel R. Tobacco, hypertension, and vascular disease: risk factors for renal functional decline in an older population. Kidney Int. 2000;57:2072-79.

49. Muntner P, Coresh J, Smith J, Eckfeldt J, Klag M. Plasma lipids and risk of developing renal dysfunction: the atherosclerosis risk in communities study. Kidney Int. 2000;58:293-301.

50. Manttari M, Tiula E, Alikoski T, Manninen V. Effects of hypertension and dyslipidemia on the decline in renal function. Hypertension. 1995;25:670-675. 
51. Yu H. Progression of chronic renal failure. Arch Intern Med. 2003;163:1417-29.

52. Erlinger T, Tarver-Carr M, Powe N, Appel L, Coresh J, Eberhardt M et al. Leukocytosis, hypoalbuminemia, and the risk for chronic kidney disease in US adults. Am J Kidney Dis. 2003;42:256-63.

53. Verma S, Anderson TJ. Fundamentals of endothelial function for the clinical cardiologist. Circulation. 2002;105:546-49.

54. Shen GX. Impact and mechanism for oxidized and glycated lipoproteins on generation of fibrinolytic regulators from vascular endothelial cells. Mol Cell Biochem. 2003;246:69-74.

55. Szekanecz Z, Koch AE. Vascular endothelium and immune responses: implications for inflammation and angiogenesis. Rheum Dis Clin North Am. 2004;30:97-114.

56. Shimokawa H. Primary endothelial dysfunction: atherosclerosis. J Mol Cell Cardiol. $1999 ; 31: 23-37$.

57. Hsueh WA, Lyon CJ, Quinones MJ. Insulin resistance and the endothelium. Am J Med. 2004;117:109-17.

58. Stenvinkel P. Endothelial dysfunction and inflammation-is there a link? Nephrol Dial Transplant. 2001;16:1968-71.

59. Calles-Escandon J, Cipolla M. Diabetes and endothelial dysfunction: a clinical perspective. Endocr Rev. 2001;22:36-52. 
60. Medvedeva, NA, Bonartsev, AP, Postnikov, AB, Slavutskaia, AV, and D'iakonov, KB. Decrease of nitric oxide (NO)-cGMP-dependent vasodilatation in the vessels of lesser circulation in endothelial dysfunction. Ross Fiziol Zh Im I M Sechenova 91(2), 132140. 2005 .

Ref Type: Journal (Full)

61. Landmesser, U, Dikalov, S, Price, SR, McCann, L, Fukai, T, Holland, SM, Mitch, WE, and Harrison, DG. Oxidation of tetrahydrobiopterin leads to uncoupling of endothelial cell nitric oxide synthase in hypertension. J Clin Invest 111(8), 1201-1209. 2003. Ref Type: Journal (Full)

62. Newsholme P, Haber EP, Hirabara SM, Rebelato EL, Procopio J, Morgan D, OliveiraEmilio HC, Carpinelli AR, and Curi R. Diabetes associated cell stress and dysfunction: role of mitochondrial and non-mitochondrial ROS production and activity. J Physiol 583(Pt 1), 9-24. 2007.

Ref Type: Journal (Full)

63. Van Zwieten P. Endothelial dysfunction in hypertension. A critical evaluation. Blood Press Suppl. 1997;2:67-70.

64. Kim J, Montagnani M, Koh K, Quon M. Reciprocal relationships between insulin resistance and endothelial dysfunction: molecular and pathophysiological mechanisms. Circulation. 2006;113:1888-904. 
65. Hein T, Singh U, Vasquez-Vivar J, Devaraj S, Kuo L, Jialal I. Human C-reactive protein induces endothelial dysfunction and uncoupling of eNOS in vivo. Atherosclerosis. 2009;206:61-68.

66. Khosla U, Zharikov S, Finch J, Nakagawa T, Roncal C, Mu W et al. Hyperuricemia induces endothelial dysfunction. Kidney Int. 2005;67:1739-42.

67. Stenvinkel P, Lindholm B, Heimburger M, Heimburger O. Elevated serum levels of soluble adhesion molecules predict death in pre-dialysis patients: association with malnutrition, inflammation, and cardiovascular disease. Nephrol Dial Transplant. 2000;15:1624-30.

68. Papayianni A, Alexopoulos E, Giamalis P, Gionanlis L, Belechri AM, Koukoudis P et al. Circulating levels of ICAM-1, VCAM-1, and MCP-1 are increased in haemodialysis patients: association with inflammation, dyslipidaemia, and vascular events. Nephrol Dial Transplant. 2002;17:435-41.

69. Landray MJ, Wheeler DC, Lip GY, Newman DJ, Blann AD, McGlynn FJ et al. Inflammation, endothelial dysfunction, and platelet activation in patients with chronic kidney disease: the chronic renal impairment in Birmingham (CRIB) study. Am J Kidney Dis. 2004;43:244-53.

70. Cottone S, Mule G, Amato F, Riccobene R, Vadala A, Lorito MC et al. Amplified biochemical activation of endothelial function in hypertension associated with moderate to severe renal failure. J Nephrol. 2002;15:643-48. 
71. Sorokin A, Kohan DE. Physiology and pathology of endothelin-1 in renal mesangium. Am J Physiol Renal Physiol. 2003;285:F579-F589.

72. Koobi P, Jolma P, Kalliovalkama J, Tikkanen I, Fan M, Kahonen M et al. Effect of angiotensin II type 1 receptor blockade on conduit artery tone in subtotally nephrectomized rats. Nephron Physiol. 2004;96:91-98.

73. Norris K, Vaughn C. The role of renin-angiotensin-aldosterone system inhibition in chronic kidney disease. Expert Rev Cardiovasc Ther. 2003;1:51-63.

74. Ruschitzka F, Shaw S, Gygi D, Noll G, Barton M, Luscher TF. Endothelial dysfunction in acute renal failure: role of circulating and tissue endothelin-1. J Am Soc Nephrol. $1999 ; 10: 953-62$.

75. Hlubocka Z, Umnerova V, Heller S, Peleska J, Jindra A, Jachymova M et al. Circulating intercellular cell adhesion molecule-1, endothelin-1 and von Willebrand factor-markers of endothelial dysfunction in uncomplicated essential hypertension: the effect of treatment with ACE inhibitors. J Hum Hypertens. 2002;16:557-62.

76. Hanson M, Gluckman P. Endothelial dysfunction and cardiovascular disease: the role of predictive adaptive responses. Heart. 2005;91:864-66.

77. Taddie S, Ghiadoni L, Virdis A, Salvetti A. Mechanisms of endothelial dysfunction: clinical significance and preventive non-pharmacological therapeutic strategies. Curr Pharm Des. 2003;9:2385-402. 
78. Taddie S, Virdis A, Ghiadoni L, Salvetti G, Bernini G, Magagna A et al. Age-related reduction of NO availability and oxidative stress in humans. Hypertension. 2001;38:274-79.

79. Cai H, Harrison D. Endothelial dysfunction in cardiovascular diseases: the role of oxidant stress. Circ Res. 2000;87:840-844.

80. Lockhart C, McVeigh G, Cohn J. Measuring endothelial function. Curr Diab Rep. 2006;6:267-73.

81. Meigs JB, Hu FB, Rifai N, Manson JE. Biomarkers of endothelial dysfunction and risk of type 2 diabetes mellitus. JAMA. 2004;291:1978-86.

82. Song Y, Manson J, Tinker L, Rifai N, Cook N, Hu F et al. Circulating levels of endothelial adhesion molecules and risk of diabetes in an ethnically diverse cohort of women. Diabetes. 2007;56:1898-904.

83. Mahmud F, Earing M, Lee R, Lteif A, Driscoll D, Lerman A. Altered endothelial function in asymptomatic male adolescents with type 1 diabetes. Congenit Heart Dis. 2006;1:98-103.

84. Thorand B, Baumert J, Chambless L, Meisinger C, Kolb H, Doring A et al. Elevated markers of endothelial dysfunction predict type 2 diabetes mellitus in middle-aged men and women from the general population. Arterioscler Thromb Vasc Biol. 2006;26:398405. 
85. Zeisler H, Livingston J, Schatten C, Tempfer C, Knöfler M, Husslein P. Serum levels of adhesion molecules in women with pregnancy-induced hypertension. Wien Klin Wochenschr. 2001;113:588-92.

86. Sahakyan K, Klein B, Myers C, Tsai M, Klein R. Novel risk factors in long-term hypertension incidence in type 1 diabetes mellitus. Am Heart J. 2010;159:1074-80.

87. Li Y, Zhang W, Wang T, Lu H, Wang Y, Wang X. The role of adhesion molecules in the pathogenic mechanisms of hypertension in obstructive sleep apnea-hypopnea syndrome. Zhonghua Jie He He Hu Xi Za Zhi. 2004;27:511-14.

88. Zhang Y, Thompson A, Tong W, Xu T, Chen J, Zhao L et al. Biomarkers of inflammation and endothelial dysfunction and risk of hypertension among Inner Mongolians in China. J Hypertens. 2010;28:35-40.

89. Ikizler TA. Nutrition, inflammation and chronic kidney disease. Curr Opin Nephrol Hypertens. 2008;17:162-67.

90. Oberg BP, McMenamin E, Lucas FL, McMonagle E, Morrow J, Ikizler TA et al. Increased prevalence of oxidant stress and inflammation in patients with moderate to severe chronic kidney disease. Kidney Int. 2004;65:1009-16.

91. Papagianni A, Dovas S, Bantis C, Belechri A, Kalovoulos M, Dimitriadis C et al. Carotid atherosclerosis and endothelial cell adhesion molecules as predictors of longterm outcome in chronic hemodialysis patients. A J Nephrol. 2008;28:265-74. 
92. Blankenberg S, Rupprecht H, Bickel C, Peetz D, Hafner G, Tiret L et al. Circulating cell adhesion molecules and death in patients with coronary artery disease. Circulation. 2001;104:1336-42.

93. Hurt-Camejo E, Paredes S, Masana L, Camejo G, Sartipy P, Rosengren B et al. Elevated levels of small, low-density lipoprotein with high affinity for arterial matrix components in patients with rheumatoid arthritis: possible contribution of phospholipase A2 to this atherogenic profile. Arthritis. 2001;44:2761-67.

94. Hanson M, Gluckman P. Endothelial dysfunction and cardiovascular disease: the role of predictive adaptive responses. Heart. 2005;91:864-66.

95. Chen N, Holmes M, Reaven G. Relationship between insulin resistance, soluble adhesion molecules, and mononuclear cell binding in healthy volunteers. J Clin Endocrinol Metab. 1999;84:3485-89.

96. Goldberg R. Cytokine and cytokine-like inflammation markers, endothelial dysfunction, and imbalanced coagulation in development of diabetes and its complications. J Clin Endocrinol Metab. 2009;94:3171-82.

97. Hwang S, Ballantyne C, Sharrett A, Smith L, Davis C, Gotto Jr A et al. Circulating adhesion molecules VCAM-1, ICAM-1, and E-selectin in carotid atherosclerosis and incident coronary heart disease cases: the Atherosclerosis Risk In Communities (ARIC) study. Circulation. 1997;96:4219-25.

98. Meigs J, Hu F, Rifai N, Manson J. Biomarkers of endothelial dysfunction and risk of type 2 diabetes mellitus. JAMA. 2004;291:1978-86. 
99. Sattar N, Murray H, Welsh P, Blauw G, Buckley B, as Craen A et al. Are elevated circulating intercellular adhesion molecule 1 levels more strongly predictive of diabetes than vascular risk? Outcome of a prospective study in the elderly. Diabetologia. 2009;52:235-39.

100. Song Y, Manson J, Tinker L, Rifai N, Cook N, Hu F et al. Circulating levels of endothelial adhesion molecules and risk of diabetes in an ethnically diverse cohort of women. Diabetes. 2007;56:1898-904.

101. Krakoff J, Funahashi T, Stehouwer C, Schalkwijk C, Tanaka S, Matsuzawa Y et al. Inflammatory markers, adiponectin, and risk of type 2 diabetes in the Pima Indian. Diabetes Care. 2006;26:1745-51.

102. Klein R, Klein BE, Linton KL, De Mets DL. The Beaver Dam Eye Study: visual acuity. Ophthalmology. 1991;98:1310-1315.

103. Linton KL, Klein BE, Klein R. The validity of self-reported and surrogate-reported cataract and age-related macular degeneration in the Beaver Dam Eye Study. Am J Epidemiol. 1991;134:1438-46.

104. Summary of revisions for the 2010 Clinical Practice Recommendation. Diabetes Care. 2010;33 Suppl 1.

105. King G. The role of hyperglycaemia and hyperinsulinaemia in causing vascular dysfunction in diabetes. Ann Med. 1996;28:427-32. 
106. Lee S, Lee D, Kim K, Lee I. Effect of estrogen on endothelial dysfunction in postmenopausal women with diabetes. Diabetes Res Clin Pract. 2001;54:S81-92.

107. Bragulat E, de la Sierra A, Antonio M, Coca A. Endothelial dysfunction in saltsensitive essential hypertension. Hypertension. 2001;37:444-48.

108. Boulanger C. Secondary endothelial dysfunction: hypertension and heart failure. J Mol Cell Cardiol. 1999;31:39-49.

109. Shimokawam H. Endothelial dysfunction in hypertension. J Atheroscler Thromb. $1998 ; 4: 118-27$.

110. Caballero A. Endothelial dysfunction in obesity and insulin resistance: a road to diabetes and heart disease. Obes Res. 2003;11:1278-89.

111. Stern MP. Diabetes and cardiovascular disease. The "common soil" hypothesis. Diabetes. 1995;44:369-74.

112. Katakam P, Ujhelyi M, Hoenig M, Miller A. Endothelial dysfunction precedes hypertension in diet-induced insulin resistance. Am J Physiol. 1998;275:R788-R792.

113. Sun X, Ku D. Selective right, but not left, coronary endothelial dysfunction precedes development of pulmonary hypertension and right heart hypertrophy in rats. Am J Physiol Heart Circ Physiol. 2006;290:H758-H764.

114. Hwang S, Ballantyne C, Sharrett A, Smith L, Davis C, Gotto Jr A et al. Circulating adhesion molecules VCAM-1, ICAM-1, and E-selectin in carotid atherosclerosis and 
incident coronary heart disease cases: the Atherosclerosis Risk In communities (ARIC) study. Circulation. 1997;96:4219-25.

115. Klein R, Klein BE, Linton KL, De Mets DL. The Beaver Dam Eye Study: visual acuity. Ophthalmology. 1991;98:1310-1315.

116. Linton KL, Klein BE, Klein R. The validity of self-reported and surrogate-reported cataract and age-related macular degeneration in the Beaver Dam Eye Study. Am J Epidemiol. 1991;134:1438-46.

117. Levey A, Coresh J, Greene T, Stevens L, Zhang Y, Hendriksen S et al. Using Standardized Serum Creatinine Values in the Modification of Diet in Renal Disease Study Equation for Estimating Glomerular Filtration Rate. Ann Intern Med. 2006;145:247-54.

118. Shimbo D, Munter P, Mann D, Viera A, Homma S, Polak J et al. Endothelial dysfunction and the risk of hypertension: the multi-ethnic study of atherosclerosis. Hypertension. Hypertension. 2010;55:1210-1216.

119. Papamichael C, Aznaouride K, Karatzis E, Stamatelopoulos K, Lekakis J, Mavrikakis M. Coffee exerts an acute unfavorable effect on endothelial function of healthy subjects. Am J Hypertension. 2004;17:74A.

120. Brook R, Brook J, Urch B, Vincent R, Rajagopalan S, Silverman F. Inhalation of fine particulate air pollution and ozone causes acute arterial vasoconstriction in healthy adults. Circulation. 2002;105:1534-36. 
121. Canty J, Schwartz J. Nitric oxide mediates flow-dependent epicardial coronary vasodilation to changes in pulse frequency but not mean flow in conscious dogs. Circulation. 1994;89:375-84.

122. Shiode N, Morishima N, Nakayama K, Yamagata T, Matsuura H, Kajiyama G. Flowmediated vasodilation of human epicardial coronary arteries: effect of inhibition of nitric oxide synthesis. J Am Coll Cardiol. 1997;27:304-10.

123. Mullen M, Kharbanda R, Cross J, Donald A, Taylor M, Vallance P et al. Heterogenous nature of flow-mediated dilatation in human conduit arteries in vivo: relevance to endothelial dysfunction in hypercholesterolemia. Circ Res. 2001;88:145-51.

124. Springer T. Traffic signals for lymphocyte recirculation and leukocyte emigration: the multistep paradigm. Cell. 1994;76:301-14.

125. Cybulsky M, Iivama K, Li H, Zhu S, Chen M, Iivama M et al. A major role for VCAM1, but not ICAM-1, in early atherosclerosis. J Clin Invest. 2001;107:1255-62.

126. Iivama K, Haira L, Iivama M, Li H, DiChiara M, Medoff B et al. Patterns of vascular cell adhesion molecule-1 and intercellular adhesion molecule-1 expression in rabbit and mouse atherosclerotic lesions and at sites predisposed to lesion formation. Circ Res. 1999;85:199-207.

127. Albelda S, Smith C, Ward P. Adhesion molecules and inflammatory injury. FASEB J. 1994;8:504-12. 
128. Gearing A, Newman W. Circulating adhesion molecules in disease. Immunol Today. 1993;14:506-12.

129. Hwang S, Ballantyne C, Sharrett A, Smith L, Davis C, Gotto AJ et al. Circulating adhesion molecules VCAM-1, ICAM-1, and E-selectin in carotid atherosclerosis and incident coronary heart disease cases: the Atherosclerosis Risk In Communities (ARIC) study. Circulation. 1997;96:4219-25.

130. Meigs JB, Hu FB, Rifai N, Manson JE. Biomarkers of endothelial dysfunction and risk of type 2 diabetes mellitus. JAMA. 2004;291:1978-86.

131. Sahakyan K, Klein B, Myers C, Tsai M, Klein R. Novel risk factors in long-term hypertension incidence in type 1 diabetes mellitus. Am Heart J. 2010;159:1074-80.

132. Cottone S, Mule G, Amato F, Riccobene R, Vadala A, Lorito MC et al. Amplified biochemical activation of endothelial function in hypertension associated with moderate to severe renal failure. J Nephrol. 2002;15:643-48.

133. Landray MJ, Wheeler DC, Lip GY, Newman DJ, Blann AD, McGlynn FJ et al. Inflammation, endothelial dysfunction, and platelet activation in patients with chronic kidney disease: the chronic renal impairment in Birmingham (CRIB) study. Am J Kidney Dis. 2004;43:244-53.

134. Stenvinkel P, Lindholm B, Heimburger M, Heimburger O. Elevated serum levels of soluble adhesion molecules predict death in pre-dialysis patients: association with malnutrition, inflammation, and cardiovascular disease. Nephrol Dial Transplant. 2000;15:1624-30. 
135. Yeun J, Levine R, Mantadilok V, Kaysen G. C-Reactive protein predicts all-cause and cardiovascular mortality in hemodialysis patients. Am J Kidney Dis. 2000;35:469-76.

136. Levey AS, Stevens LA, Schmid C, Zhang Y, Castro 3rd A, Feldman H et al. A New Equation to Estimate Glomerular Filtration Rate. Ann Intern Med. 2009;150:604-12.

137. Verma S, Anderson TJ. Fundamentals of endothelial function for the clinical cardiologist. Circulation. 2002;105:546-49.

138. Shen GX. Impact and mechanism for oxidized and glycated lipoproteins on generation of fibrinolytic regulators from vascular endothelial cells. Mol Cell Biochem. 2003;246:69-74.

139. Szekanecz Z, Koch AE. Vascular endothelium and immune responses: implications for inflammation and angiogenesis. Rheum Dis Clin North Am. 2004;30:97-114.

140. Shimokawa H. Primary endothelial dysfunction: atherosclerosis. J Mol Cell Cardiol. $1999 ; 31: 23-37$.

141. Caballero AE. Endothelial dysfunction in obesity and insulin resistance: a road to diabetes and heart disease. Obes Res. 2003;11:1278-89.

142. Hsueh WA, Lyon CJ, Quinones MJ. Insulin resistance and the endothelium. Am J Med. 2004;117:109-17.

143. Stenvinkel P. Endothelial dysfunction and inflammation-is there a link? Nephrol Dial Transplant. 2001;16:1968-71. 
144. Papayianni A, Alexopoulos E, Giamalis P, Gionanlis L, Belechri AM, Koukoudis P et al. Circulating levels of ICAM-1, VCAM-1, and MCP-1 are increased in haemodialysis patients: association with inflammation, dyslipidaemia, and vascular events. Nephrol Dial Transplant. 2002;17:435-41.

145. Janssen U, Ostendorf T, Gaertner S, Eitner F, Hedrich HJ, Assmann KJ et al. Improved survival and amelioration of nephrotoxic nephritis in intercellular adhesion molecule-1 knockout mice. J Am Soc Nephrol. 1998;9:1805-14.

146. Kawasaki K, Yaoita E, Yamamoto T, Tamatani T, Miyasaka M, Kihara I. Antibodies against intercellular adhesion molecule-1 and lymphocyte function-associated antigen-1 prevent glomerular injury in rat experimental crescentic glomerulonephritis. J Immunol. $1993 ; 150: 1074-83$.

147. Norman M, James W, Hickey M. Differential roles of ICAM-1 and VCAM-1 in leukocyte-endothelial cell interactions in skin and brain of MRL/faslpr mice. J Leukoc Biol. 2008;84:68-76.

148. Perticone F, Maio R, Perticone M, Sciacqua A, Shehaj E, Naccarato P et al. Endothelial dysfunction and subsequent decline in glomerular filtration rate in hypertensive patients. Circulation. 2010;122:379-84.

149. American Heart Association, Cardiovascular Disease Statistics. American Heart Association. 2010. 
150. Song Y, Manson J, Tinker L, Rifai N, Cook N, Hu F et al. Circulating levels of endothelial adhesion molecules and risk of diabetes in an ethnically diverse cohort of women. Diabetes. 2007;56:1898-904.

151. de Lemos J, Hennekens C, Ridker P. Plasma concentration of soluble vascular cell adhesion molecule-1 and subsequent cardiovascular risk. J Am Coll Cardiol. 2000;36:423-26.

152. Klein R, Klein BE, Linton KL, De Mets DL. The Beaver Dam Eye Study: visual acuity. Ophthalmology. 1991;98:1310-1315.

153. Linton KL, Klein BE, Klein R. The validity of self-reported and surrogate-reported cataract and age-related macular degeneration in the Beaver Dam Eye Study. Am J Epidemiology. 1991;134:1438-46.

154. Al-Lsa A, Thalib L, Akanji A. Circulating markers of inflammation and endothelial dysfunction in Arab adolescent subjects: reference ranges and associations with age, gender, body mass and insulin sensitivity. Atherosclerosis. 2010;208:543-49.

155. Lennon P, Collard C, Morrissey M, Stahl G. Complement-induced endothelial dysfunction in rabbits: mechanisms, recovery, and gender differences. Am J Physiol. 1996;270:H1924-H1932.

156. Ravikumar R, Deepa R, Shanthirani C, Mohan V. Comparison of carotid intima-media thickness, arterial stiffness, and brachial artery flow mediated dilatation in diabetic and nondiabetic subjects (The Chennai Urban Population Study [CUPS-9]). Am J Cardiol. 2002;90:702-7. 
157. Wong W, Wong S, Tian X, Huang Y. Endothelial Dysfunction: The Common Consequence in Diabetes and Hypertension. J Cardiovasc Pharmacol. 2010;55:300-307.

158. Goch A, Banach M, Mikhailidis D, Rysz J, Goch J. Endothelial dysfunction in patients with noncomplicated and complicated hypertension. Clin Exp Hypertens. 2009;31:2030.

159. Bessa S, Hamdy S, El-sheikh R. Serum visfatin as a non-traditional biomarker of endothelial dysfunction in chronic kidney disease: an Egyptian study. Eur J Med. 2010;21:530-535.

160. Cottone S, Palermo A, Arsena R, Riccobene R, Guarneri M, Mule G et al. Relationship of fetuin-A with glomerular filtration rate and endothelial dysfunction in moderatesevere chronic kidney disease. J Nephrol. 2010;23:62-69.

161. Martos R, Valle M, Morales R, Canete R, Gascon F, Urbano M. Changes in body mass index are associated with changes in inflammatory and endothelial dysfunction biomarkers in obese prepubertal children after 9 months of body mass index SD score loss. Metabolism. 2009;58:1153-60.

162. Villela N, Aguiar L, Bahia L, Bottino D, Bouskela E. Does endothelial dysfunction correlate better with waist-to-hip ratio than with body mass index or waist circumference among obese patients? Clinics. 2006;61:53-58.

163. Yeun J, Levine R, Mantadilok V, Kaysen G. C-Reactive protein predicts all-cause and cardiovascular mortality in hemodialysis patients. Am J Kidney Dis. 2000;35:469-76. 